\title{
Disc-corona energetics in the Very High State of Galactic Black Holes
}

\author{
Chris Done ${ }^{1}$ and Aya Kubota ${ }^{2}$ \\ ${ }^{1}$ Department of Physics, University of Durham, South Road, Durham DH13LE, UK; chris.done@durham.ac.uk \\ ${ }^{2}$ Cosmic Radiation Laboratory, Institute of Physical and Chemical Research, \\ 2-1 Hirosawa, Wako-shi, Saitama, 351-0198, Japan; aya@crab.riken.jp
}

Accepted $2005 * * *$. Received $2005 * * *$; in original form $2005 * * *$

\begin{abstract}
The X-ray spectra of Galactic binary systems dominated by a quasi-thermal component (disc dominated or high/soft state) are well described by a standard ShakuraSunyaev disc structure down to the last stable orbit around the black hole. This is not the case in the very high (or steep power law) state, where the X-ray spectra show both a strong disc component and strong, steep tail to higher energies. We use simultaneous optical-ASCA-RXTE data from the black hole transient XTE J1550-564 as a specific example of two such spectra, where the power emitted in the tail is more than 50 per cent of the bolometric luminosity. These have disc spectra which are significantly lower in temperature than those seen from the same source at the same luminosity in the high/soft state. If these give a true picture of the disc then either the disc emissivity has reduced, and/or the disc truncates above the last stable orbit.

However, it is often assumed that the tail is produced by Compton scattering, in which case its shape in these spectra requires that the Comptonising region is marginally optically thick $(\tau \sim 2-3)$, and covers a large fraction of the inner disc. This will distort our view of the disc, especially of the hottest temperature material. We build a theoretical model of a Comptonising corona over an inner disc, and fit this to the data, but find that it still requires a large increase in inner disc radius for a standard disc emissivity. Instead it seems more probable that the disc emissivity changes in the presence of the corona. We implement the specific inner disc-corona coupling model of Svensson \& Zdziarski (1994), in which some fraction $f$ of the accretion power is dissipated in the corona leaving only a fraction $1-f$ to be dissipated in the optically thick disc. We show that this can explain the low temperature/high luminosity disc emission seen in the very high state with only a small increase in radius of the disc. While this inferred disc truncation is probably not significant given the model uncertainties, it is consistent with the low frequency QPO and gives continuity of properties with the low/hard state spectra.
\end{abstract}

\section{Key words:}

accretion, accretion discs - black hole physics - radiation mechanisms: thermal radiative transfer - X-rays: binaries - X-rays: individual: XTE J1550 - 564

\section{INTRODUCTION}

Galactic black hole binaries at high mass accretion rates show spectra which are often dominated by a soft, quasithermal component (e.g. the review by Tanaka \& Lewin 1995). This shape matches very well with that expected from an optically thick, geometrically thin accretion disc, as described in the seminal paper by Shakura \& Sunyaev (1973). This identification is strengthened by observations of variability in which the temperature, $T_{i n}$, and luminosity, $L$, of this quasi-thermal component change together in such a way as to indicate that the size of the emitting structure remains approximately constant $\left(L \propto T_{i n}^{4}\right)$. The last stable orbit is the only obvious candidate for such a radius, so this gives compelling evidence for a key prediction of general relativity, as well as providing an observable diagnostic of the mass and spin of the black hole (Ebisawa et al 1994; Kubota et al 2001; Gierliński \& Done 2004; Kubota \& Done 2004;

(c) 0000 RAS 
hereafter KD04). The most recent models of accretion disc spectra are able to reproduce this behavior (Davis et al 2005; Davis, Done \& Blaes 2006). These supersede earlier models which showed strong deviations from the $L \propto T_{i n}^{4}$ relation, but which did not include metal opacity nor treat the difference in vertical structure between the radiation and gas pressure dominated regimes (Merloni, Fabian \& Ross 2000).

The disc dominated spectra always contain some small fraction of emission in a tail extending to much higher energies. However, some high luminosity spectra have a rather different spectral shape, where the tail contains a much larger fraction of the bolometric luminosity. The disc emission in these spectra (variously termed very high or steep power law dominant state, hereafter VHS; Miyamoto et al. 1991; McClintock \& Remillard 2003) is less obvious due to the lower contrast against the strong continuum. Nonetheless, it can be identified in spectral fitting, but its derived parameters (temperature and luminosity) are somewhat dependent on how the tail is modelled, which in turn depends on the radiation processes involved. A power law or broken power law description for the tail might be appropriate if the emission is from nonthermal electrons in the jet, but these models require fine tuning in order to match all the observational constraints. Conversely, Comptonisation models, where the disc is the source of seed photons, rather naturally reproduce both multiwavelength spectral constraints and X-ray variability patterns (see $\S 3$ ), so here we explore the consequences of such models.

Firstly, the disc temperature in GBH is close to (or within!) the observed X-ray bandpass, so the low energy break in the Comptonised component is predicted to be present, giving a rather different spectrum at low energies to that of a power law which extends unbroken through the disc emission. Thus using proper Comptonisation models can significantly change the parameters of the inferred disc component (Done, Życki \& Smith 2002) and distort the measured luminosity-temperature relation (Kubota et al 2001; Kubota \& Makishima 2004; KD04). This helps considerably in interpreting data from the weak VHS, where the disc contributes more than half of the total bolometric luminosity. A power law model for the tail in these data gives derived disc temperatures which are significantly higher than those seen from the same source at the same disc luminosity in the high/soft state. Using proper Comptonisation models instead of the power law approximately recovers the $L \propto T_{i n}^{4}$ behavior (Kubota et al 2001; Kubota \& Makishima 2004; KD04). The match to the high/soft state data is even better with an additional correction to the observed disc luminosity to account for the photons which are scattered into the Comptonised spectrum (Kubota \& Makishima 2004; KD04).

However, the most strongly Comptonised VHS (where the disc is less than half of the bolometric luminosity) have disk spectra which show significantly lower temperatures than those seen at the same disc luminosity in the high/soft state. These are inconsistent with a constant disc radius even with proper Comptonisation models and correction for scattering (KD04). This could indicate that the disc is truncated above the last stable orbit (KD04), but here we explore whether this conclusion is required with a more sophisticated approach to Comptonisation. The parameters derived for the hot electrons indicate that the Compton cloud is marginally optically thick, with $\tau \sim 2-3$. This material plainly must intercept the disc photons in order to Comptonise them, so will also distort our view of the disc (KD04). An optically thick corona over the inner disc could simply hide the hottest part of the disc from view. More likely, the power dissipated in the corona can reduce the disc emissivity (e.g. Svensson \& Zdziarski 1994) since the energy in the corona must derive ultimately from the accretion flow. We build explicit models of an inner disc- corona to explore these ideas, and fit them to two strong VHS spectra (simultaneous $0.7-200 \mathrm{keV}$ ASCA and RXTE data) of the Galactic black hole binary XTE J1550 - 564 to constrain the possible geometries of the disk-corona in this state.

In $\S 2$, we review the spectral parameters (luminosity and temperature) of the geometrically thin disk in the disk dominated high/soft state. In $\S 3$ we describe the simultaneous ASCA-RXTE observations of the broadband spectra. We compare the derived disc emission in the VHS to that seen in the high/soft state, showing that it implies a very different disc area and/or emissivity ( $§ 4)$. In $\S 5$ we describe our model of an inner disc corona above a standard emissivity disc. Fitting this to the data shows explicitly that optical depth effects alone cannot hide the inner disc, and that this model requires a large change in disc area compared to the high/soft state. We modify this in $\S 6$ to incorporate energetic coupling of the inner disc and corona as in Svensson \& Zdziarski (1994). The reduction in disc emissivity and temperature under the corona means this model can fit the observed spectra with only a small - or possibly no - increase in disc area.

We review all the results of the models in $\S 7$, and then incorporate additional constraints from other spectral and timing characteristics of this state in $\S 8$. We show that an optically thick Comptonisation in an inner disc corona is not inconsistent with the presence of high frequency QPO's. However, it does conflict with claims of a strong, extremely broad iron line in these spectra (Miller et al 2004). We show that there is strong evidence supporting Comptonisation as the emission mechanism for the tail ( $(9)$, whereas there is an alternative explanation for the iron line, in which its width is overestimated due to the unrecognized presence of absorption lines (Done \& Gierlinśki 2006).

All the Comptonisation models considered here require that the inner disc changes in extent and/or emissivity in response to the presence of the corona. There are no solutions in which the inner disc has the same radial structure as that seen in the high/soft state.

\section{THE DISK DOMINANT HIGH/SOFT STATE AS A TEMPLATE FOR THE VHS GEOMETRY}

In all the following we use the binary system parameters determined by the optical studies of Orosz et al. (2002) i.e. mass of $8.4-11.2 M_{\odot}$, distance, $D$, of $\sim 5 \mathrm{kpc}$ and binary inclination angle, $i$, of $70^{\circ}$ (Orosz et al. 2002). However, we are primarily interested in changes in the disc emitting area rather than absolute values i.e. in comparing the disc structure in the VHS with that seen in the disc dominated high/soft states. The high/soft states in XTE J1550 - 564 have been well fit using the DISKBB model (Mitsuda et al. 1984) in XSPEC (Kubota \& Makishima 2004; Gierlińksi \& 
Done 2004; KD04), giving a clear $L \propto T_{i n}^{4}$ relationship, implying constant area, over a factor 100 change in disc luminosity. Such an obviously thermal relationship between luminosity and temperature from DISKBB fits is now predicted by the best current models of the disc spectra which include radiative transfer with full metal opacities and self consistent vertical structure as well as relativistic effects (Davies et al 2005).

The apparent inner disc radius of this source, $r_{\mathrm{in}}$, is obtained from the normalization of DISKBB model, defined as $r_{\text {in }}^{2} \cos i /(D / 10 \mathrm{kpc})^{2}$. The average value inferred from all the high/soft state constant area DISKBB results is $59 \mathrm{~km}$. However, the 'true' inner disc radius is related to this apparent radius via a series of correction factors (for the stress free inner boundary condition, effective temperature not equal to colour temperature and relativistic effects; e.g. Kubota et al 1998; 2001). However, the fractional change in inferred disc radius is unaffected by uncertainties in distance, inclination and correction factors, so we give the apparent inner radius, $r_{\text {in }}$ as a ratio of DISKBB normalisation seen in these fits to that derived from all the high/soft state data in Kubota \& Makishima (2004). These ratios are not sensitive to any of the binary system uncertainties, so can sensitively show the change in the disc properties between the high/soft and VHS.

\section{ASCA- $R X T E$ SIMULTANEOUS OBSERVATIONS}

ASCA observations of this source were performed three times, on 1998 September 12, 23, and 1999 March 17. There are simultaneous $R X T E$ observations corresponding to each ASCA observation. The second data set shows a strongly Comptonised VHS spectrum, and was included in the analysis of KD04. Here we also study the first simultaneous data set, which is at the boundary between the low/hard state and the strongly Comptonised VHS. We do not use the third ASCA dataset as the gain was changed on the corresponding RXTE data dataset midway through the observation, and the spectrum is only a weakly comptonised VHS. The observational ID's of the corresponding RXTE data are 30188-06-05-00 and 30191-01-10-00, for the first and second ASCA observations, respectively. We use the data as extracted by KD04 (standard procedures for selecting good PCA and HEXTE data, with PCA responses derived for each observation using pcarsp v8.0: see Appendix for a comparison with pcarsp v10.1). We use the PCA data from 3$20 \mathrm{keV}$, and HEXTE from 20-200 keV.

The ASCA GIS events were extracted from circular regions of $6^{\prime}$ radius centered on the image peak, after selecting good time intervals in a standard procedure. Net exposures of $1.4 \mathrm{ks}$ and $2.4 \mathrm{ks}$ were obtained for the simultaneous pointing on the first and second observations, respectively. The dead time correction fractions for the first ASCA data set are $76.1 \%$ for GIS2 and $77.9 \%$ for GIS3, determined by reference to the count-rate monitor data with little dead time, using the method described in Makishima et al (1996). Similarly, for the second observation, these are calculated as $85.6 \%$ and $87.3 \%$ for GIS2 and GIS3, respectively. We only use the GIS data $(0.7-10 \mathrm{keV})$ since the SIS is strongly affected by pileup.
In order to take into account the calibration uncertainties, we add $1 \%$ systematic errors to the GIS spectra (Makishima et al 1996) and $0.5 \%$ systematic errors to the PCA. We also include $0.5 \%$ systematic uncertainty on the HEXTE data to be conservative, though this does not significantly change the fit (see Appendix). We allow $N_{H}$ to be free (described using WABS) as the low energy bandpass of the ASCA GIS constrains this parameter. The data from different instruments are fitted with same model parameters except that relative normalization factors are free. Hereafter, we use the normalization of the PCA in the fitting results so as to be able to directly compare with the PCA analysis of Kubota \& Makishima (2004) for the disc spectra. Using this we find the observed (i.e. absorbed) $0.7-200 \mathrm{keV}$ fluxes of $7.2 \times 10^{-8} \mathrm{erg} \mathrm{s}^{-1} \mathrm{~cm}^{-2}$ and $8.3 \times 10^{-8} \mathrm{erg} \mathrm{s}^{-1} \mathrm{~cm}^{-2}$ for the first and second observations, respectively, corresponding to observed luminosities of $2.2 \times 10^{38} \mathrm{erg} \mathrm{s}^{-1}$ and $2.5 \times 10^{38} \mathrm{erg} \mathrm{s}^{-1}$ for isotropic emission. Correcting for absorption and the energy band gives a bolometric luminosity of $\sim 4.0$ and $4.7 \times 10^{38} \mathrm{erg} \mathrm{s}^{-1}$ i.e. about 35 per cent of the Eddington limit.

\section{CONTINUOUS DISC-CORONA}

\subsection{Fitting the data}

These VHS spectra are dominated by a strong, steep continuum rather than the disc, though the disc can still be seen. The continuum shape is complex: fits using a single power law (together with the disc, and including a smeared edge and Gaussian to phenomenologically model the reflection components) are completely statistically unacceptable, with $\chi_{\nu}^{2}$ of 2538/195 and 1718/195, for the first and second datasets, respectively. This was also shown by Sobczak et al (1999), who had to fit the PCA and HEXTE data separately in order to get acceptable fits with a power law continuum. However, the continuum curvature is complex: a single thermal Comptonisation model for the tail (with disc, smeared edge and Gaussian line) gives a much improved fit over a power law, but the fits are not formally acceptable at $\chi_{\nu}^{2}$ of $402.9 / 193$ and 261.5/193, respectively.

The complex form of the tail is shown in more detail by Gierliński \& Done (2003), who used OSSE data together with the PCA and HEXTE to extend the bandpass beyond $500 \mathrm{keV}$ for times around that of the second dataset used here. These clearly showed that the curvature seen in the tail cannot be matched by Comptonisation from thermal or non-thermal electrons (see Fig. 5 of Gierliński \& Done 2003). Instead the continuum shows features of both thermal and non-thermal electrons (see also Zdziarski et al 2001 for similar spectra from GRS 1915+105). Here we follow KD04 and approximate this by using the THCOMP model (Zdziarski, Johnson \& Magdziarz 1996; now publically available as a local model in XSPEC ${ }^{\star}$ ) to describe the thermal Compton scattering, together with a weak power law of $\Gamma$ fixed at 2 to approximate the higher energy (non- thermal) emission. We have checked that our results on the disc temperature and luminosity are not dependent on this approximation

\section{$\star$}

http://heasarc.gsfc.nasa.gov/docs/xanadu/xspec/newmodels.html 


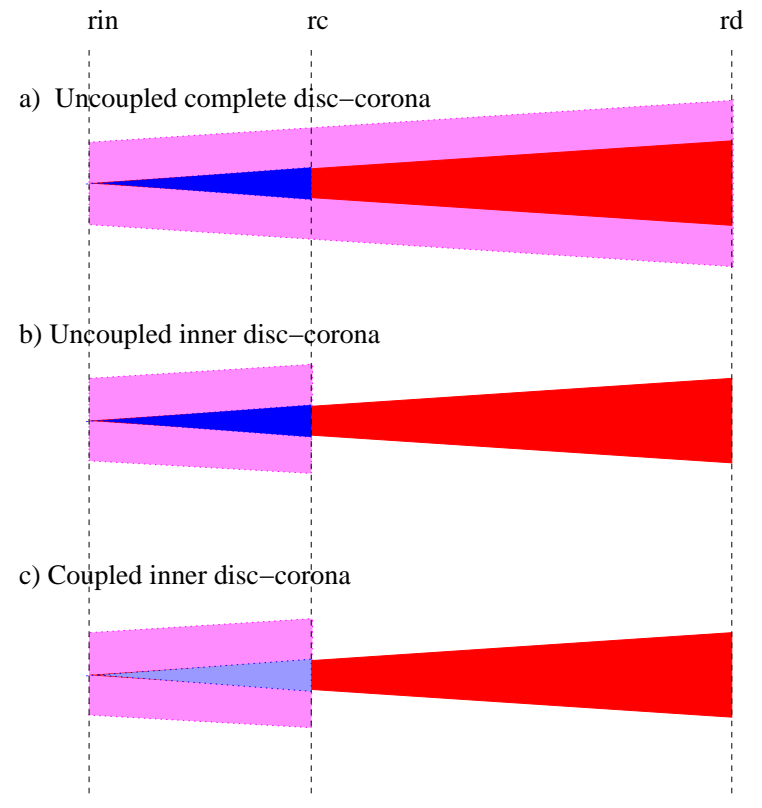

rd
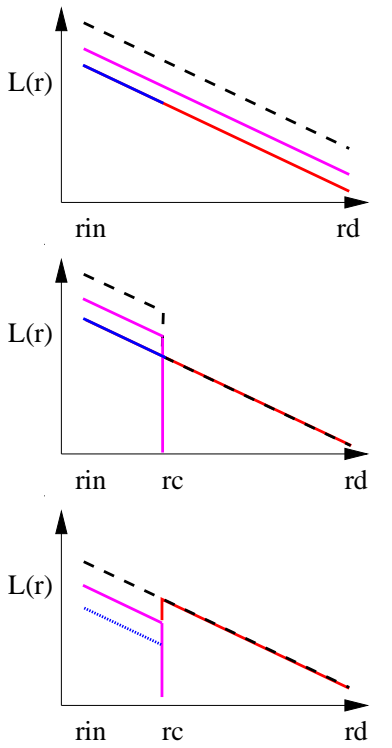

Figure 1. The schematic geometry envisaged for each of the models described in the text. To the right of each geometry is a sketch of the associated emissivity as a function of radius for the outer disc (red), inner disc (blue), corona (magenta) and total (black) accretion flow.

for the shape of the high energy spectrum by using the full hybrid thermal-nonthermal Comptonisation code EQPAIR (Coppi 1999) on our final model of the Comptonised disc-corona described in $\S 6$.

The seed photons in THCOMP are set to be from the disc (giving a slightly different low energy rollover to that produced by the COMPTT model in the XSPEC general release which assumes a Wien distribution). This is equivalent to assuming that the Comptonising corona covers the whole disc, as shown schematically in Fig. 1a.

Following KD04 we use several different descriptions of the reflected emission, to try to assess the effect on the derived disc parameters of systematic uncertainties in current reflection models. Firstly we use the Gaussian line and smeared edge to give a phenomenological description of reflection (SEG). Then we replace these components with the physical reflection model incorporated in THCOMP (REF) which includes both full reflected continuum and selfconsistent iron line emission for arbitrary ionization state of the reflected material (Życki, Done \& Smith 1999). Ionization is important as the lack of photoelectric opacity in ionized material means reflection contributes to the low energy spectrum so can affect the derived disc temperature and luminosity.

However, the ionised reflection in THCOMP does not include Compton up and downscattering of the reflected emission in the disc itself. This is important for highly ionized and/or hot discs, and leads to strong distortions on the predicted line and edge shape in the reflected emission which can affect the spectral fitting, either by distorting the inferred solid angle of reflecting material, or its ionization or amount of relativistic smearing (Ross, Fabian \& Young 1999; Ballantyne, Ross \& Fabian 2001; Done \& Gierlińksi 2006).

Fitting with better ionized reflection models which do include the Compton scattering on the line and edge is not straightforward as these models are tabulated for an illuminating power law continuum, which is not appropriate for these data. Hence we attempt to quantify the effect of these systematic model uncertainties in the reflected emission by removing the 5-12 keV region (i.e. the iron line and edge features which are sensitive to the disc Comptonisation) from the spectral fits. We then refit the spectra with ionization of the reflector fixed at $10^{2}(\mathrm{Xi} 2), 10^{3}(\mathrm{Xi} 3)$ and $10^{4}(\mathrm{Xi} 4)$ in order to assess how much the derived disc luminosity and temperature are dependent on this parameter, and so get an estimate of the systematic model uncertainties (as in KD04).

We tabulate results from all free parameters in these fits in Table A1 in the Appendix. Uncertainties are given for $\Delta \chi^{2}=2.7$, and derived from a full error scan where all other parameters are free to vary. We include the derived disc luminosity, which is also subject to uncertainties in distance and inclination, but we stress that this is used only to compare with the disc luminosity derived using the same distance and inclination. The reflected fraction is also dependent on the assumed inclination, but this is not used further in the paper.

Fig. 2a and b shows each $\nu F_{\nu}$ spectrum, deconvolved and corrected for absorption using the best fit SEG model (phenomenological reflection), together with residuals. The two spectra are qualitatively similar, with the disc emission being strongly Comptonised. The only differences are that the uncomptonised disc spectrum is slightly easier to identify in the first spectrum than in the second, and the spectral index of the Comptonised emission is slightly flatter. This immediately sets some constraints on the Comptonising geometry. The corona will scatter only a fraction $C_{f}[1-\exp (-\tau)]$ where $C_{f}$ is the covering fraction of the corona over the disc and $\tau$ is the optical depth. Thus if either $C_{f}$ or $\tau$ were small then the disc spectrum would be above the Comptonised tail. Since it is not, the spectra in 

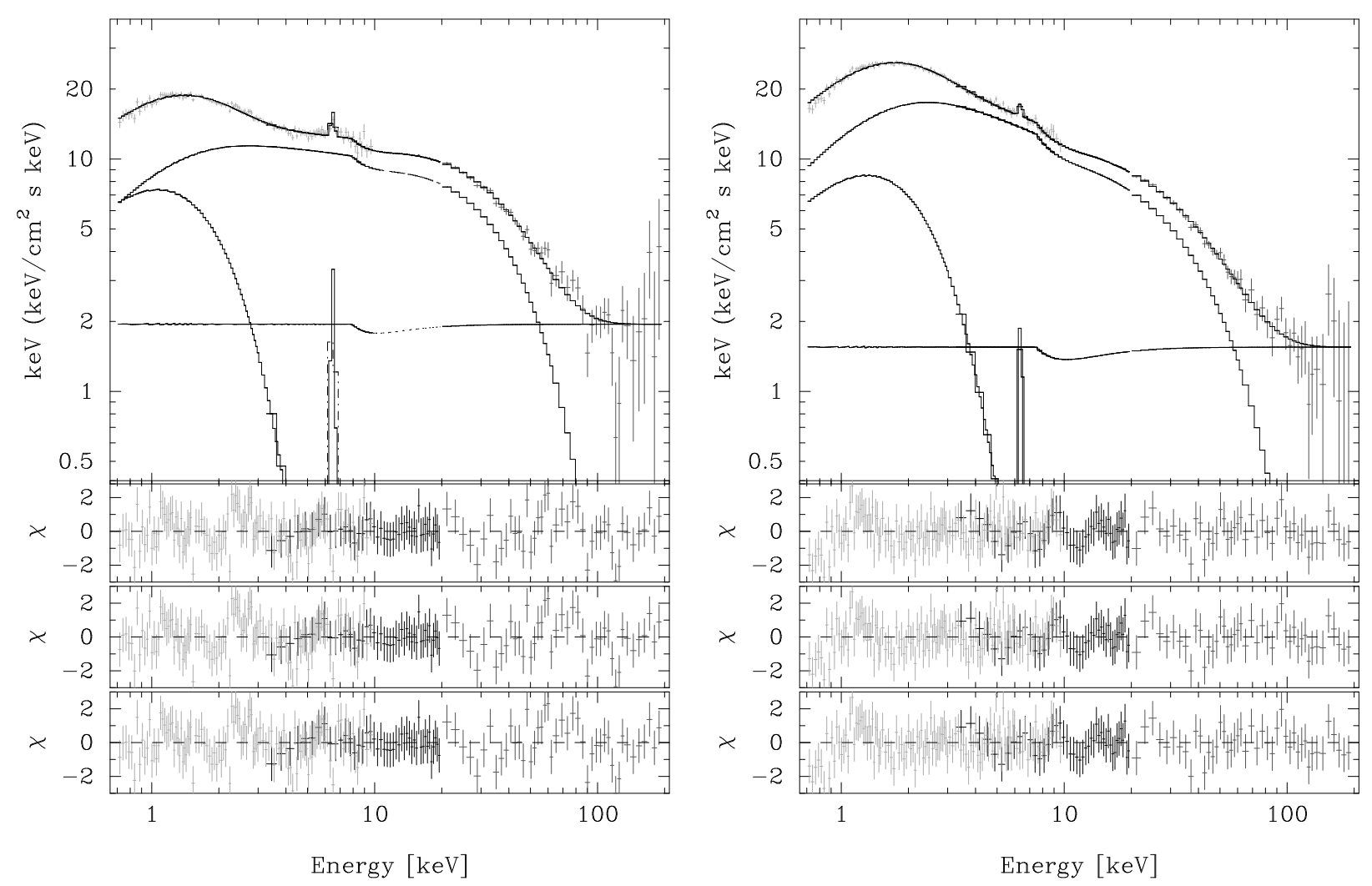

Figure 2. Absorption corrected intrinsic spectra for each of the two observations described in the text. The left and right hand panels show the simultaneous ASCA-RXTE data taken before and after the peak, respectively. Both datasets are deconvolved using the best fit phenomenological model with SEG reflection, and the ASCA and HEXTE normalisations are set to match the PCA. The residuals are from this fit (SEG in Table A1), the DKвBтн fits (SEG in Table A2) and the DKBвFTh fits (SEG in Table A3), respectively, showing that all these different disc-corona models are a good description of the overall spectral shape.

Fig. $2 \mathrm{a}$ and $\mathrm{b}$ show that the majority of the disc emission is covered by an optically thick corona (see also the discussion in KD04).

Both spectra also show two clear breaks within the bandpass of the data, a high energy downturn at $\sim 30 \mathrm{keV}$ and a low energy downturn at $1.2 \mathrm{keV}$ and $1.8 \mathrm{keV}$ for the first and second datasets, respectively. We stress that the low energy downturn seen in these data are not due to absorption, since the spectra in these figures are absorption corrected, though it is dependent on the assumption that the continuum seed photons are from the disc. Our derived column of $\sim 0.7 \pm 0.2 \times 10^{22} \mathrm{~cm}^{-2}$ is consistent within uncertainties with that of $0.8 \pm 0.2 \times 10^{22} \mathrm{~cm}^{-2}$ derived from Chandra data (Miller et al 2003) and is slightly less than the full column through our Galaxy in this direction $\left(0.9 \times 10^{22}\right.$ $\mathrm{cm}^{-2}$ ), as expected.

The existence of the low and high energy cutoffs within the observed $0.7-200 \mathrm{keV}$ bandpass mean that these data cover the majority of the accretion power output. Thus the total luminosity is well defined (modulo uncertainties in the distance and inclination which cancel when comparing to the disc luminosity derived from high/soft state data), rather than being strongly affected by uncertainties associated with extrapolating the spectral models outside of the observed range.

\subsection{Derived luminosity and temperature of the disc emission}

Fig 3 shows the luminosity and temperature for the disc dominated high/soft state observations of KD04 to compare with those seen from the disc in the two VHS state spectra used here. Reconstructing the intrinsic disc emission is model dependent: a Comptonising corona will distort the observed disc spectrum. However, the Comptonised spectrum itself contains a low energy rollover, directly imprinting the energy of the seed photons on the boosted emission. Thus with broad bandpass spectra, where the energy range of the expected disc temperature is covered by the observations as in these ASCA-RXTE data, the intrinsic seed photon temperature is recoverable.

The seed photon luminosity is equally observable, despite the effects of Comptonisation shrouding the disc. Compton scattering increases the photon energy by on average $\Delta E / E \sim 4 \tau^{2}\left(k T_{\mathrm{e}} / m_{e} c^{2}\right)$. Thus the Comptonised luminosity is related to the scattered disc luminosity simply by a factor $\sim(1+\Delta E / E) \sim 1.3$ for the steep Comptonised spectra observed here. This scattered disc luminosity should simply be added to the observed disc luminosity to estimate the intrinsic disc luminosity of $\left(L_{\text {disc }}+L_{\text {thcomp }}\right) / 1.3$ Thus the intrinsic disc luminosity cannot be very much smaller than the total luminosity, which is robustly estimated by these data. This is shown plotted against observed disc tempera- 
(a)diskbb+thcomp

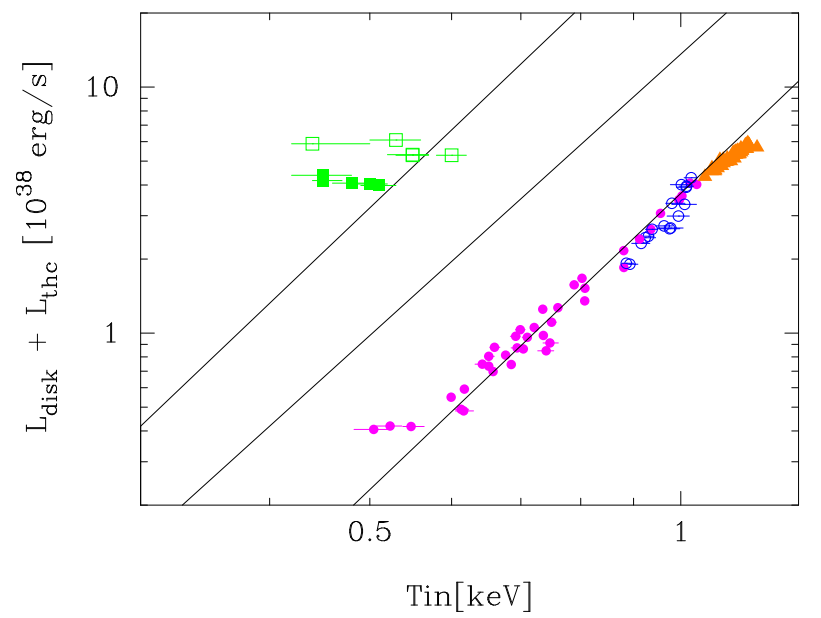

(c)dkbbth

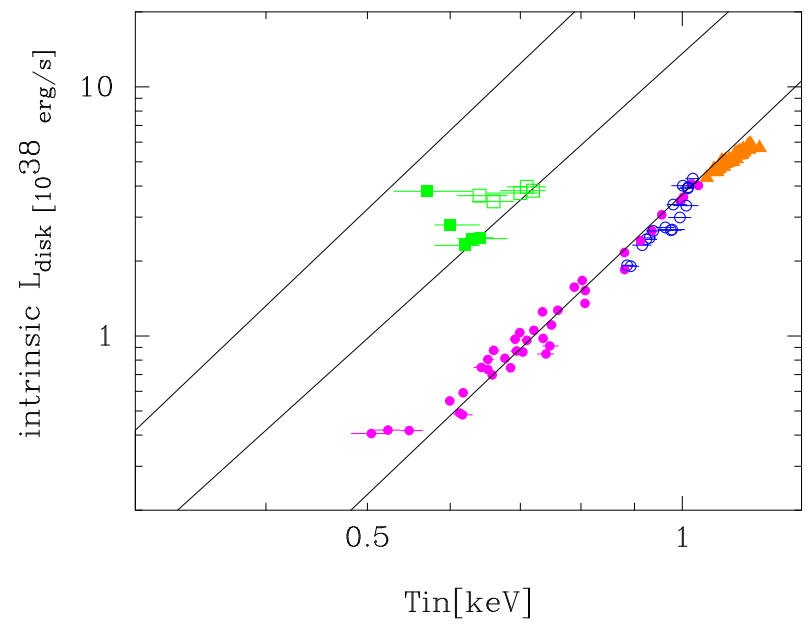

(b) diskbb+thcomp

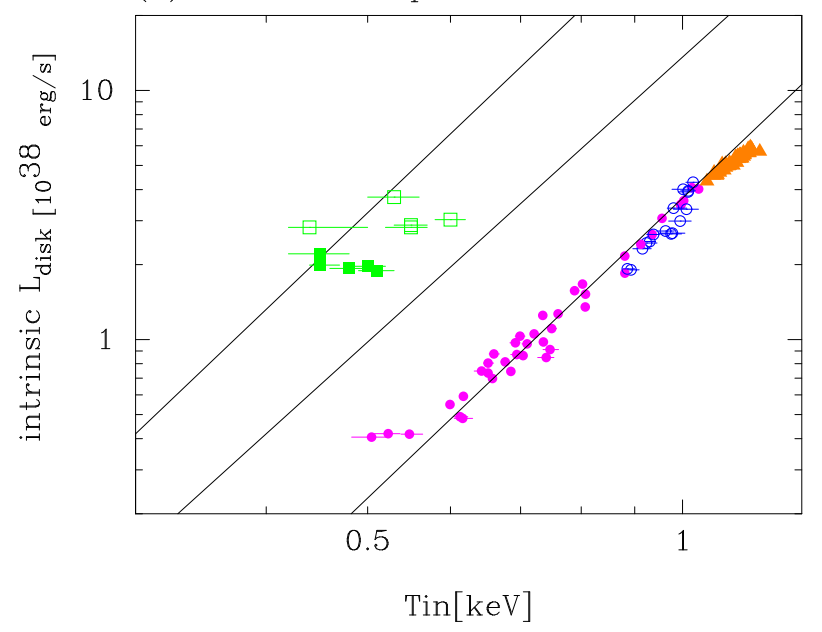

(d) dkbbfth

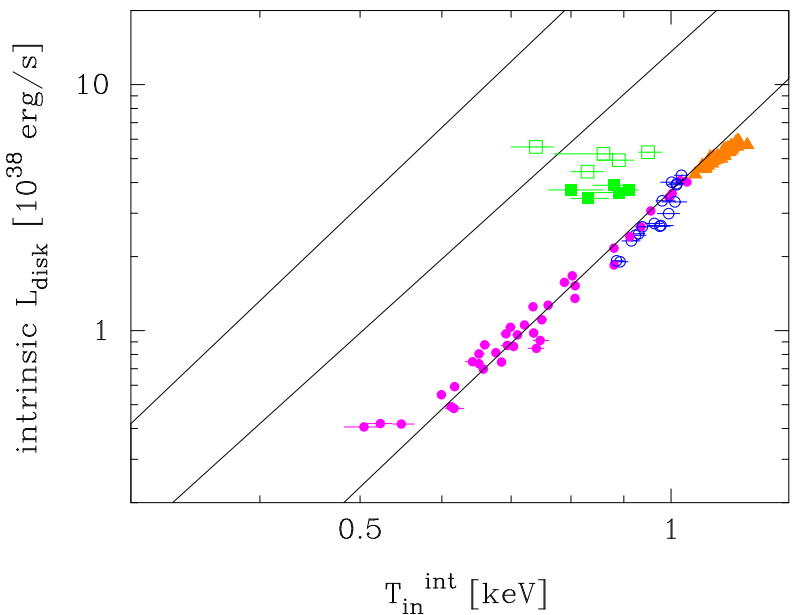

Figure 3. Luminosity versus temperature plots for the disc. Filled circles and filled triangles show the data from the disc dominated states, while the open circles show the weakly comptonised very high state (KD04). Filled and open squares and open squares show the strongly comptonised very high state data from before and afer the peak, respectively. There are five points for each of these strongly comptonised spectra, corresponding to the five different reflection descriptions. The solid lines show $L \propto T^{4}$ for $r_{\text {in }}=59,100$ and $200 \mathrm{~km}$. Panel a and b show the reconstructed disc emission from simple modelling of the spectra (Table A1), corresponding to a corona covering the whole disc (see Fig. 1a). Panel a plots the observed disc temperature against the inferred disc luminosity estimated by $L_{d i s c}+L_{t h c o m p}$. This is a slight overestimate since Comptonisation boosts the seed photon luminosity by a factor $\sim 1.3$ for these data. Panel $\mathrm{b}$ shows a more careful reconstruction of the disc luminosity using the photon numbers and inclination of the slab. Either way gives similar results, showing that the inferred disc luminosity and temperature is quite robustly estimated by these data, and that they imply the inner edge of the disc is much larger than that seen in the disc dominated spectra. Panel c shows the reconstructed inner disc temperature and luminosity for an inner disc-corona (Fig. 1b). This is not very different to that found from the previous fits. By contrast, Panel d shows the results for an inner disc whose energetics are coupled to that of the corona (Fig. 1c). The implied change in inner disc radius is now much smaller.

ture in Fig 3a, and plainly shows a marked discrepancy with the constant radius/constant colour temperature correction observed from the disc dominated spectra.

A more careful correction for the effects of Compton scattering can be done by noting that this conserves photon number so the intrinsic disc luminosity can be simply estimated by increasing the observed disc luminosity by a factor $\left(a N_{c o m p}+N_{\text {disc }}\right) / N_{\text {disc }}$ where $N_{\text {comp }}$ and $N_{\text {disc }}$ are the numbers of photons in the thermally Comptonised and disc spectral components, respectively, and $a$ are $2 \cos i$ and 1 for spherical and slab geometries (see Kubota \& Makishima 2004; KD04). Panel b shows the reconstructed disc luminosity assuming a slab geometry and clearly shows that the inferred disc is very different to that seen in the high/soft state data.

Thus, while spectral fitting is almost always non-unique, the presence of the low and high energy rollover in the observed bandpass mean that we can make very robust esti- 
mates for the intrinsic disc temperature and luminosity, giving a marked discrepancy with the constant radius/constant colour temperature correction observed from the disc dominated spectra. Disc dominated spectra at the same luminosity have a temperature of $\sim 1 \mathrm{keV}$, so for a spectrum dominated by Comptonisation we expect a low energy rollover from the seed photon energy at $4 k T_{\text {in }} \sim 4 \mathrm{keV}$. This is in direct conflict with the observation of the low energy rollover at $1.2 \mathrm{keV}$ or $1.8 \mathrm{keV}$ which shows the seed photon temperature is $<0.5 \mathrm{keV}$ or $<0.7 \mathrm{keV}$. The inferred disc is highly luminous but with low temperature, implying that the inner radius of the disc is a factor $\sim 3$ higher in the VHS than in the disc dominated spectra.

Plainly, with these models the disc is not consistent with a constant inner radius, constant emissivity, constant colour temperature correction between the VHS and the disc dominated state. At least one of these must be changing. An obvious change is for the corona to affect the colour temperature correction. Irradiation will change the vertical temperature structure of the disc, but this should lead to an increasing temperature for the upper layers, so an increased colour temperature correction. Yet the data show a lower temperature for the inferred luminosity. Similarly, conduction between the corona and disc would also increase the disc temperature, as would an increase in the stress at the inner disc boundary (Agol \& Krolik 2000). This robust discrepancy between the disc structure in the disc dominated and VHS motivates the more sophisticated modelling in the rest of the paper.

\section{INNER DISC CORONA, SEPARATE ENERGETICS}

Here we build a simple model of an inner disc corona, and fit it to the data, incorporating the effects of the geometry as well as the radiative constraints from Comptonisation. The corona is assumed to exist only at small radii (see Fig 1b) so it removes only the hottest part of the disc spectrum by Comptonising it, while not affecting the spectrum of the cooler disc at larger radii. The aim is to test whether this more physically realistic model still requires the inferred increase in disc inner radius seen in the previous section.

\subsection{Models}

We assume that the corona is a slab of constant optical depth and temperature on top of the disc, extending from $r_{\text {in }}$ to $r_{\mathrm{c}}$, while the disc itself extends from $r_{\text {in }}$ to $1000 r_{\text {in }}$ (see Fig. 1b). Each radius in the disc is assumed to emit blackbody radiation, with the standard temperature distribution of a multicolour DISKBB i.e. $T(r)=T_{\text {in }}\left(r / r_{\text {in }}\right)^{-3 / 4}$. Where the disc is not covered by the Compton slab then its emission is unaffected. Otherwise, it is suppressed by a factor $\exp (-\tau)$, where $\tau$ is determined from the Comptonised spectrum using the observed $\Gamma$ and $k T_{\mathrm{e}}$, assuming a slab geometry. The blackbody emission from the disc at this radius is used as the seed photons for the Comptonising cloud, whose spectrum is then normalised so that the total number of photons in the Comptonised spectrum is equal to the number of photons scattered from the blackbody disc emission at that radius i.e. $1-\exp (-\tau)$.
A more careful treatment would have to account for the different angle dependences of the seed photons and comptonised emission - the number of seed photons which emerge unscattered is more accurately $\exp (-\tau / \cos i)$, but the number which are scattered into this direction is not simply $1-\exp (-\tau / \cos i)$ as photons scattered into a given line of sight come from a range of initial seed photon angles (see e.g. Pozdnyakov, Sobol \& Sunyaev 1983). There is yet more complexity as about half the Compton scattered photons will be directed down towards the disc. Those which are not reflected will be reprocessed, adding to the intrinsic disc emission (Haardt \& Maraschi 1993). We neglect all these effects in order to be able to approximately calculate the spectrum.

The shape of the spectrum is determined by the parameters of the Compton corona $\left(\Gamma\right.$ and $k T_{\mathrm{e}}$ ), the size of this corona relative to the disc $r_{\mathrm{c}} / r_{\mathrm{in}}$, and intrinsic inner disc temperature, $k T_{\text {in }}$. The overall normalisation gives the apparent disc radius, $r_{\mathrm{in}}$, for the assumed distance and inclination, as in the DISKBB model. Even though the full DISKBB spectrum is not seen, the model normalisation is that of the uncomptonised disk. The model parameters for the disk component are in effect what the disk would have looked like without the Comptonising corona, and so can be directly compared with the disk normalisation derived from the disk dominant high/soft state.

Fig. 4a shows the results for this model corona parameters close to those implied by the VHS data i.e. a corona with $k T_{\mathrm{e}}=10 \mathrm{keV}$ and $\tau=2$ (which corresponds to a photon index of the scattered flux of $\Gamma=2.3$ as the optical depth for a slab geometry is half of that which gives the same spectrum for a sphere). The inner disc temperature is set to $1 \mathrm{keV}$ to match that seen from the disc dominated spectra at similar luminosities (see Fig. 3). The radius of the Comptonising region is progressively increased over more and more of the disc, from $r_{\mathrm{c}} / r_{\text {in }}=1,1.33,1.66,3.33$ and 1000. This changes the spectrum from a pure disc blackbody, to a more complex shape. While this does have the same number of free parameters as the previous model, it is somewhat more constrained. The normalisation of the disc and Comptonised components is not a completely free parameter, but is set by $r_{\mathrm{c}} / r_{\text {in }}$ and by the assumed slab geometry. In the limit where the corona covers all the disc then the spectrum has the same shape as that of a THCOMP model with disc blackbody seed photons but with the addition of $\exp (-\tau)$ of the initial disc blackbody spectrum which is unscattered by the corona.

The spectrum always shows the imprint of the seed photon energy at $\sim 1 \mathrm{keV}$. This is because of the physical requirements of the model, firstly that the seed photons are from the inner disc with standard emissivity, and secondly that the Comptonised spectrum has the same number of photons in it as were removed from the disc emission. A standard disc emissivity disc produces most of its luminosity in the inner regions, and it is these inner regions which are covered by the corona. The Compton energy boost is not large for these coronal parameters $\left(\Gamma=2.3, k T_{\mathrm{e}}=10 \mathrm{keV}\right)$ so the Comptonised photons, with their clear seed photon rollover, add to the emission at similar energies to those of the seed photons. Comptonisation conserves photon number, so the normalisation of the spectrum is also similar. Thus the optically thick corona does intercept most of the 

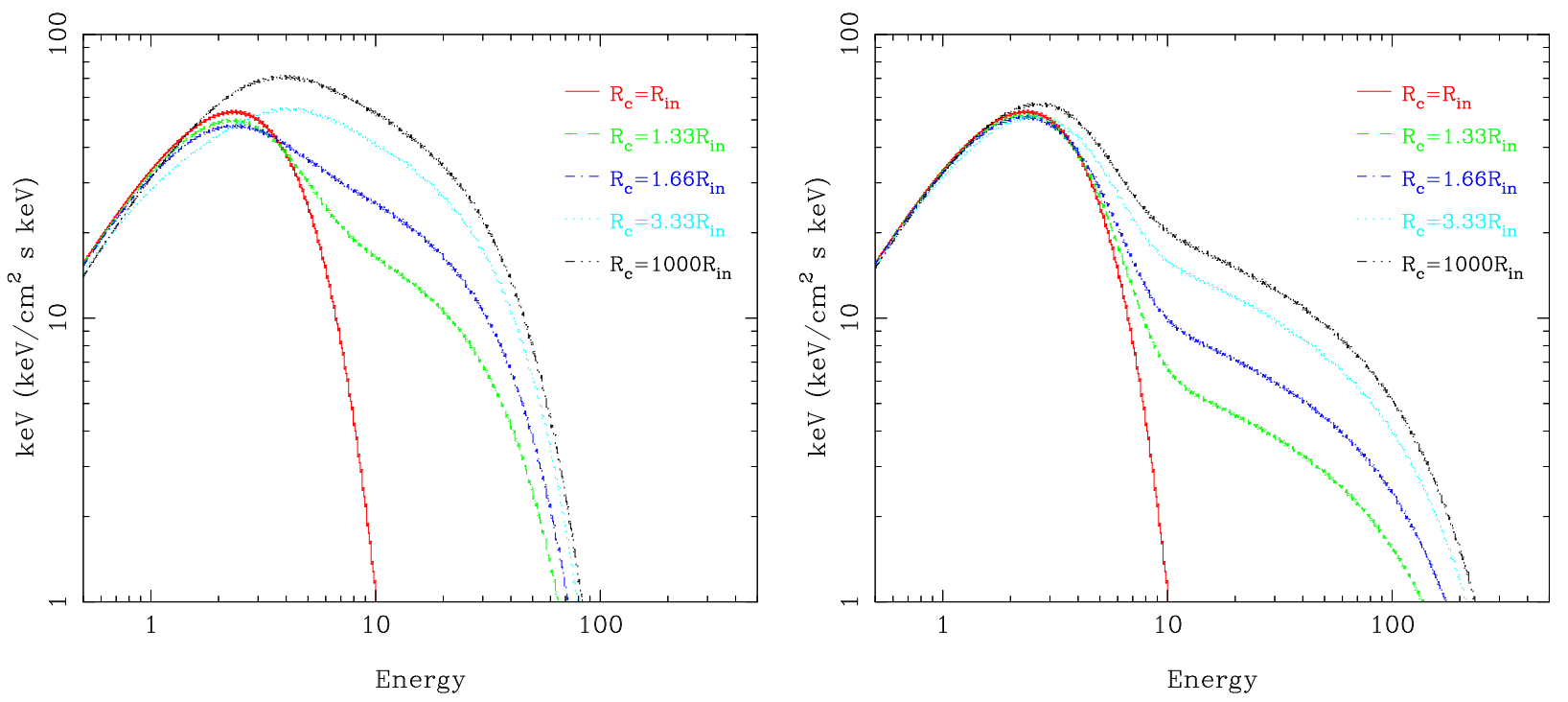

Figure 4. An inner disc corona model for $r_{\mathrm{c}}=1$ (red, i.e. equivalent to no corona), 1.33 (green), 1.66 (blue), 3.33 (cyan) and 1000 (black i.e. equivalent to a corona which completely covers the disc) $\times r_{\text {in }}$ (see Fig. 1b). The left panel shows the results for coronal parameters close to those inferred from the data, with $\Gamma=2.3$ and $k T_{\mathrm{e}}=10 \mathrm{keV}$. This corresponds to $\tau \sim 2$ for a slab corona. The right panel shows the effect of increasing the coronal temperature to $100 \mathrm{keV}$. To keep the same spectral index for higher temperature means that the optical depth has to decrease to $\tau \sim 0.4$. Thus there is always a clear unscattered disc component (unlike the data), and the intrinsic disc temperature of $1 \mathrm{keV}$ is seen.

inner disc emission, but this Comptonisation does not hide it as neither the energy nor number of photons are changed substantially.

Figure $4 \mathrm{~b}$ shows the same series of spectra for increasing coronal radius, but with $k T_{\mathrm{e}}=100 \mathrm{keV}$. Though increasing the electron temperature increases the energy boost so making it possible to move the photons away from their seed energy, to get the same spectral index of $\Gamma=2.3$ from this higher temperature plasma requires a lower optical depth. The corona becomes optically thin so the spectrum now is clearly always two component (disc plus Comptonisation), and the disc is always also clearly at $1 \mathrm{keV}$. Although only the inner disc photons are now boosted far away from their seed photon energy, the low optical depth means that not enough of them are Comptonised to hide the hottest disc components even for a corona that extends over the whole disc.

A comparison by eye of these models with the observed spectra in Fig. 2 shows that the observed low energy rollover at $\sim 1.8 \mathrm{keV}$ strongly constrains the seed photon energy even in an inner disc corona model to be substantially less than $1 \mathrm{keV}$. Thus this model is not going to easily change the conclusion of the previous section that the inner disc temperature is much lower than is seen at this luminosity in the disc dominated high/soft states. These models of an inner disc corona above a standard emissivity disc still require that the disc inner radius in the VHS is substantially larger than in the high/soft disc dominated states.

\subsection{Data fitting}

We quantify the argument above by replacing the separate DISKBB and THCOMP components in the spectral fits with this new inner disc corona model. We include the THCOMP reflection model in the disc-corona code, to take into account illumination of the outer disc by the Comptonised spectrum. Again we use the same range of ionized reflection descriptions as before in order to quantify the uncertainties which arise from our modeling of this component.

First we fit this model to the simultaneous ASCARXTE spectra with the coronal radius fixed at $r_{\mathrm{c}} / r_{\mathrm{in}}=$ 1000. This model has one less free parameter than the separate DISKBB+ THCOMP fits shown in $\S 4$ because of the specific geometry (slab corona) assumed. The observed spectral shape of the Comptonised emission ( $\Gamma$ and $k T_{\mathrm{e}}$ ) set $\tau$ for the specific geometry, and this fixes the ratio of normalisations of disc and Comptonised emission. The fits are significantly worse, showing that our assumed specific continuous slab corona geometry is not consistent with the data. However, the inferred disc temperatures for the underlying standard emissivity DISKBB spectrum are $\sim 0.48 \mathrm{keV}$ and $0.51 \mathrm{keV}$ for the first and second data set, respectively. These are much the same temperatures as for the previous fits (though with worse $\chi^{2}$ due to the assumed geometry), illustrating the robustness of reconstruction of the seed photon (intrinsic disc) temperature

We then repeat the fits with the coronal radius as a free parameter to correspond to the geometry of Fig.1b (again with all five different reflection descriptions). While this formally gives the same number of degrees of freedom as the separate DISKBB+THCOMP fits, the model is more constrained by the geometric and physical assumptions. Nonetheless, the fits are indistinguishable in $\chi^{2}$ from those in $\S 4$, strongly supporting an inner disc-corona geometry for these VHS spectra. Table A2 gives the results for the underlying (i.e. intrinsic, before Comptonisation) DISKBB temperature and luminosity. These are plotted in the middle panel of Fig. 3b, together with the points from the disc dominated 

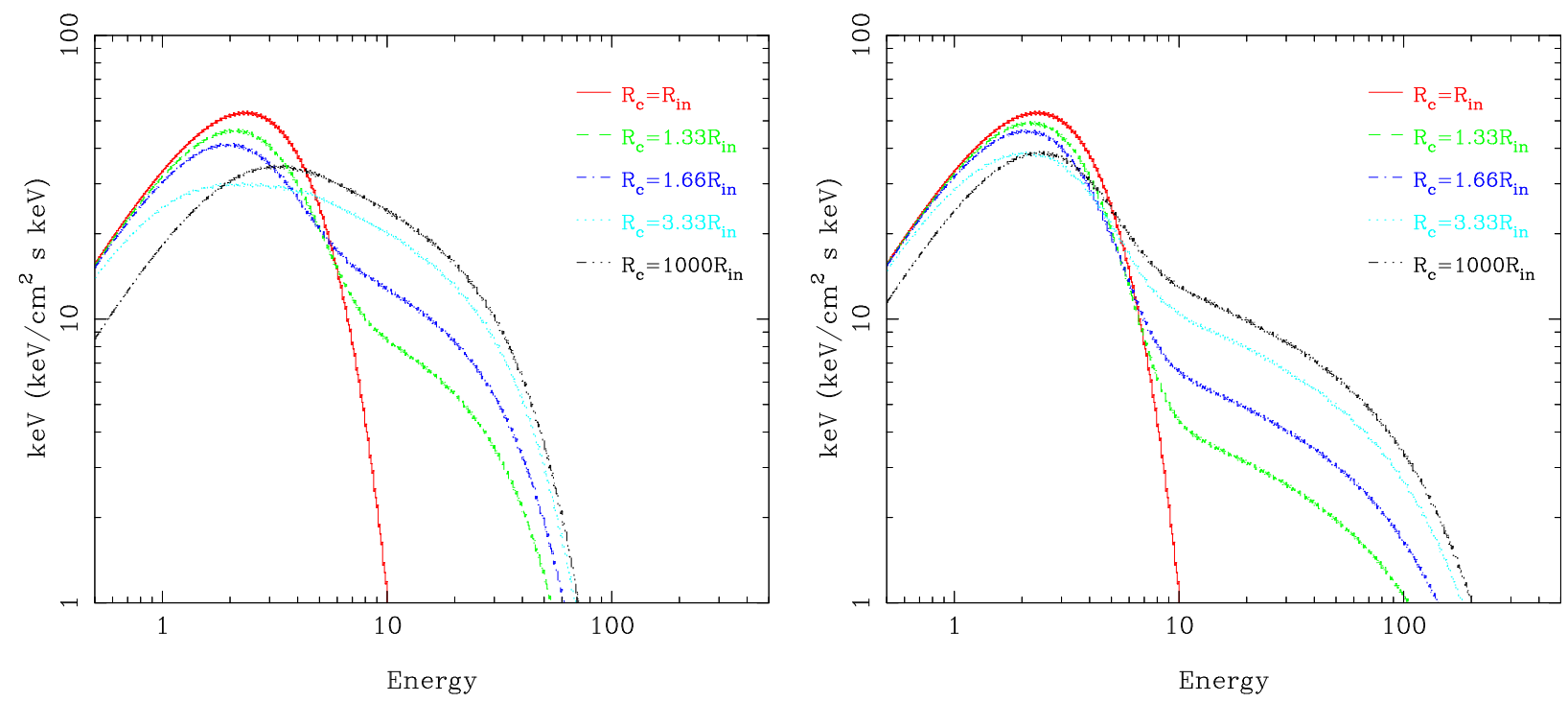

Figure 5. As in Fig.4, but for an inner disc-corona with coupled energetics (see Fig. 1c). The spectra left hand panel $(\Gamma=2.3$, $\left.k T_{\mathrm{e}}=10 \mathrm{keV}\right)$ have $f \sim 0.5$ for $r<r_{\mathrm{c}}$, while those in the right hand panel $\left(\Gamma=2.3, k T_{\mathrm{e}}=100 \mathrm{keV}\right)$ have $f \sim 0.3$.

spectra (KD04). Plainly, as shown above, the ASCA-RXTE spectra still strongly require a very low temperature disc which does not fall on the constant radius/constant colour temperature correction line derived for the disc dominated spectra. Again the inferred apparent inner disc radius is a factor 2 larger than that seen in the high/soft disc dominated states.

\section{INNER DISC CORONA, COUPLED ENERGETICS}

The previous model assumed that the disc emission is unaffected by the presence of the corona. That is, the inner disc corona model assumes that while the disc radiates efficiently at all radii, the coronal mass flow only radiates within $r_{\mathrm{c}}$. However, this seems rather artificial. The corona dissipates some power, and this must derive ultimately from the accretion flow. The disc and coronal flows could be kept separate in a continuous corona geometry (e.g. Fig. 1a) with some (approximately constant) fraction of the power and/or mass accretion taking place via the corona (Życki et al 1997, Haardt \& Maraschi 1993; Svensson \& Zdziarski 1994). The geometry of an inner disc-corona instead implies that there is a single accretion flow at large radii, $\dot{M}_{\mathrm{disc}, \infty}$ which then splits at $r_{\mathrm{c}}$ to form the disc and corona (Fig.. 1c). Again, we build a model incorporating these concepts, and fit it to the data, to test whether it still requires an increased inner disc radius.

\subsection{Models}

We use the specfic disc-corona coupling model of Svensson \& Zdziarski (1994), which assumes most of the mass accretion rate is still carried by the disc, but where a fraction $f$ of the accretion power is dissipated in the corona. The power dissipated in the optically thick disc is discontinuous at $r_{\mathrm{c}}$, where it drops from $\dot{M}_{d i s c, \infty}$ by a factor $(1-f)$. This implies the disc temperature is also discontinuous, dropping to $(1-f)^{1 / 4}$ of that expected from $\dot{M}_{d i s c, \infty}$ (see Svensson \& Zdziarski (1994) calculate the effect on the disc in the limit in which most of the mass accretion rate is still carried by the disc, but where a fraction $f$ of the accretion power is dissipated in the corona. The power dissipated in the optically thick disc is discontinuous at $r_{\mathrm{c}}$, where it drops from $\dot{M}_{d i s c, \infty}$ by a factor $(1-f)$. This implies the disc temperature is also discontinuous, dropping to $(1-f)^{1 / 4}$ of that expected from $\dot{M}_{d i s c, \infty}$. This is shown schematically in Fig. 1c.

We incorporate these ideas into our inner disc-corona code to predict the spectrum from a coupled disc-corona system. The code starts with the standard emissivity disk to produce an initial guess at the seed photon luminosity and temperature and calculates the Comptonised flux as before (using $\Gamma$ and $k T_{\mathrm{e}}$ to determine $\tau$, and scattering a fraction $1-\exp (-\tau)$ of the disc photons into the Comptonised emission). The total Comptonised flux from all radii is used to calculate a (radially averaged) value of $f$ which is used to recalculate the disk emissivity and temperature, acting as seed photons for the Comptonising corona, giving a slightly different value of $f$. The code iterates once more around this loop in order to reach a self-consistent solution. The parameters of the model are the same as for the uncoupled inner disccorona system described above. Again, the inner disc temperature is that of the uncomptonised disk i.e. the DISKBB which would have been seen without the corona present. For the uncoupled disk-corona this is simply the underlying disc emission, whereas for the coupled disk-corona the underlying disc emission is distorted from that of a DISKBB by the effect of the corona draining energy from the disk. The 'temperature' which parameterizes this coupled disk-corona systems is the innermost disc temperature, $T_{\text {in }}^{\text {int }}$, which would be seen in the limit of $f=0$, not the inner disc temperature used in making the model which is $(1-f)^{1 / 4} T_{\mathrm{in}}^{\mathrm{int}}$.

Fig. 5a and b shows the spectra obtained from such models with the same intrinsic $\operatorname{disc}\left(T_{\mathrm{in}}^{\mathrm{int}}=1 \mathrm{keV}\right)$ and 

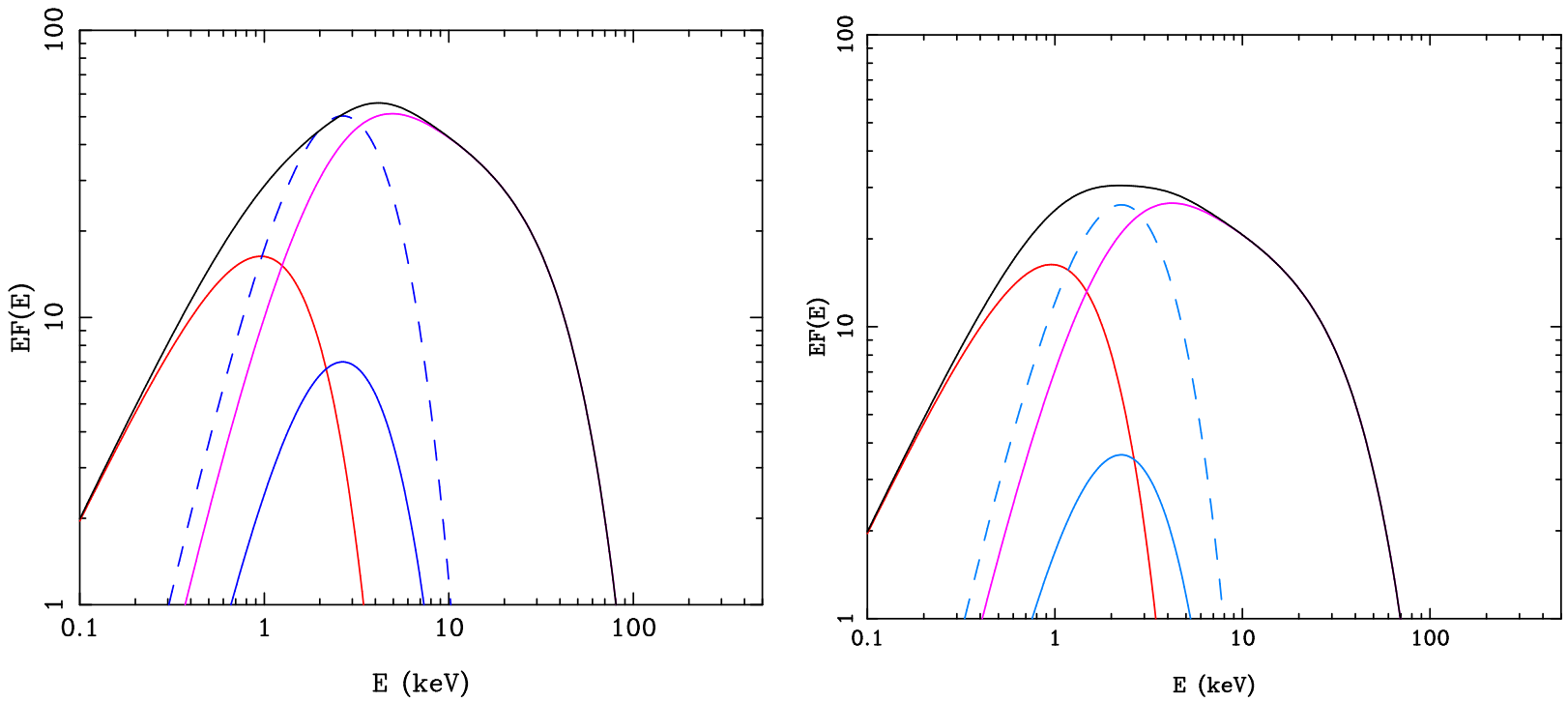

Figure 6. The model spectra for $\Gamma=2.3$ and $k T_{\mathrm{e}}=10 \mathrm{keV}$ for $r_{\mathrm{c}} / r_{\mathrm{in}}=3.3$. The total spectrum (black) is the sum of the contribution from the outer disc (red), uncomptonised inner disc (blue line), and comptonised emission (magenta). The dashed blue line shows the intrinsic (before Comptonisation) inner disc emission. The left panel shows the uncoupled disc corona, while the right panel shows the coupled system. There is a marked decrease in luminosity in the coupled system due to the assumed emissivity (compare the right hand panels of Fig. 1b and c), but more importantly there is also a marked decrease in the low energy rollover seen in this spectrum. This is mostly due to the decrease in emissivity of the inner disc emission. This lowers the normalisation of the comptonised spectrum, so increasing the relative contribution of the low temperature, outer disc.

coronal parameters as for the separate disc-corona shown in Fig. 4a (where $\Gamma=2.3, k T_{e}=10 \mathrm{keV}$, implying $f \sim 0.5$ in the corona) and $\mathrm{b}$ (where $\Gamma=2.3, k T_{e}=100 \mathrm{keV}$, implying $f \sim 0.3$ in the corona).

Fig. 6 illustrates the differences between the coupled and uncoupled coronae by showing the individual spectral components for a corona with $\Gamma=2.3, k T_{e}=10 \mathrm{keV}$ and $r_{\mathrm{c}} / r_{\text {in }}=3.3$. Both spectra have the same $\dot{M}_{\text {disk, } \infty}$, and assume the same inner disc radius (so without the corona both would give the same disc temperature of $T_{\text {in }}=T_{\text {in }}^{\text {int }}=1 \mathrm{keV}$ ) but there is much less total power in the coupled disc-corona system. This is as expected, as there is the additional coronal emissivity in the uncoupled disc-corona model. More importantly, the low energy rollover in the spectrum which is used to indicate the seed photon temperature is much lower in the coupled disc-corona. The reason for this is not primarily due to the reduction in temperature of the inner accretion flow under the corona. This is only a factor $(1-f)^{1 / 4}$ lower than $T_{\text {in }}^{\text {int }}$ i.e. $0.85 \mathrm{keV}$, so the low energy rollover in the Comptonised spectra is not significantly smaller $\left(4 k T_{\mathrm{in}}=3.4 \mathrm{keV}\right.$ as opposed to $4 \mathrm{keV}$; KD04, magenta line). The major reason for the lower energy rollover in the total temperature is the reduction in emissivity of the inner disc in the coupled corona system. The dotted blue lines show the intrinsic inner disc emission, while the solid blue lines show the emergent, uncomptonised disc emission. The intrinsic disc emission is a factor $(1-f)$ weaker in the coupled disc-corona, so the Comptonised spectrum is also a factor $(1-f)$ weaker. A standard emissivity DISKBB has maximum luminosity from the inner disc components, hence the Comptonised emission (with its high seed photon rollover temperature) is generally dominant. For the coupled disc-corona, the reduction in emissivity under the corona means that the luminosity of the corona can be less than the luminosity of the outer disc. The Comptonised emission in the coupled system still contains a fairly high seed photon rollover, at $(1-f)^{1 / 4} T_{\mathrm{in}}^{\mathrm{int}}$, but this can be masked by the much lower temperature rollover from the outer disc emission at $\sim T_{\text {in }}\left(r_{\mathrm{c}} / r_{\text {in }}\right)^{-3 / 4} \sim 0.4 \mathrm{keV}$. The major effect of the coupled energetics is to decrease the fraction of energy emitted in the inner disc-corona, so enhancing the relative importance of the cooler, outer disc emission.

\subsection{Data Fitting}

We refit the data with this coupled disc-corona model, in which the disc emissivity changes from that of a standard disc. Again we use all 5 different descriptions of reflection to quantify the effect of systematic uncertainties in spectral modelling. Details of the fits to the data are given in Table A3, and again the models give equally as good a description of the data as either the uncoupled inner disc/slab corona (§ 5), or the continuous disc/corona system (§ 4).

The most obvious change from the coupled energetics is that the inferred inner disc radius is much smaller than in the previous fits. This is due to the same combination of two effects as given above $(\S 6.1)$ i.e. firstly and most importantly the increased relative strength of the low temperature emission from the outer (uncomptonised) disc, and secondly the slight reduction in temperature of the inner disc due to the energy drained from it to power the corona. The right hand panel of Fig. 3 shows the the derived luminosity and temperature of the disc without its corona, $T_{\mathrm{in}}^{\mathrm{int}}$, compared to the high/soft state luminosity-temperature data. The VHS points now lie much closer to those from the disc dominated states than before (left and middle panels of Fig. 3) though formally the statistical uncertainties show that the 
$20 \%$ increase in disc radius in the VHS is significant (assuming a constant colour temperature correction). However, this is probably completely compatible with a constant disc radius given the systematic modelling uncertainties e.g. our assumptions about the shape of the corona (slab, with constant $\tau$ and $T_{e}$ ), the form of disc-corona coupling (all $\dot{M}$ through the disc, as opposed to some fraction of the accretion also taking place via the corona) and neglect of selfconsistent reprocessing of the illuminating coronal flux by the disc.

\section{SUMMARY OF SPECTRAL FITS}

The VHS spectra seen in some high luminosity Galactic black holes are plainly strongly affected by Compton scattering. The most extreme VHS spectra no longer have a clear disc component, separate from the Compton scattered flux, but instead show a smooth continuum shape. The clear observational inference is that the radii which produce the majority of the disc luminosity must be covered by optically thick material.

Assuming the inner disc is covered by an optically thick corona obviously makes it difficult to observe the properties of the inner disc, though the intrinsic disc luminosity and temperature can still be reconstructed from broad bandpass spectra. The temperature can be determined from the low energy rollover in the Compton scattered spectrum, while the disc luminosity is related to the Compton scattered luminosity simply through the energy boost from Comptonisation.

We use the two simultaneous ASCA-RXTE (0.7$200 \mathrm{keV}$ ) observations of the extremely Comptonised VHS spectra from XTE J1550 - 564 to investigate the intrinsic disc emission. As shown by KD04, this emission is not consistent with that seen in the disc dominated state from the same object. The temperature is much lower than that seen during the disc dominated states of the same luminosity.

We build a simple model of a slab corona over a disc to show that this conclusion holds irrespective of whether the corona covers the whole disc, or only the inner portion. The data strongly require that either the disc radius has increased, or the disc emissivity has decreased (or both). We sketch each of these possible VHS geometries in Fig. 7a$c$, respectively. If the disc does truncate, the accretion flow should still extend down to the last stable orbit unless there is a powerful outflow (wind/jet) at this point. Thus we show a continuous corona inward of the truncated disc as being the likely geometry in Figs.7a and c.

The one common denominator for all the geometries in Figs. 7 is that the disc is strongly affected by the Comptonising corona. The coronal flow is not some separate phase of accreting material, e.g. a low angular momentum flow. Instead it must couple in some way to the disc.

\section{ADDITIONAL CONSTRAINTS FROM THE DATA}

There is additional information in the spectral and variability properties of these data which can give further constraints on the geometry. Firstly, the overall shape of the

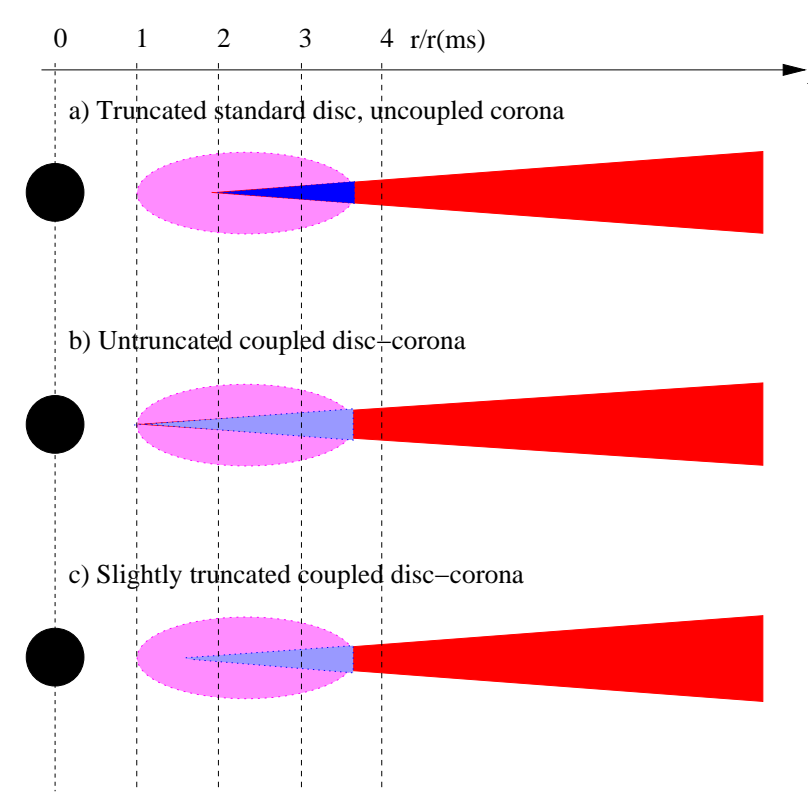

Figure 7. Possible geometries for the VHS, with radii for the disc and corona in a) and c) taken from the average of the Xi1, Xi2 and $\mathrm{Xi} 3$ model fits to the spectrum after the peak with DKBBTH (Table A2) and DквBfтн (Table A3), respectively.

Comptonised spectrum is steep $(\Gamma>2)$, so the average energy gain is rather small. This means that the seed photon luminosity is similar to the luminosity emitted by the electrons i.e. to the heating rate of the electrons. This is easy to arrange in the corona over the disc since the disc provides copious seed photons. However, it is much more difficult in the geometry of Fig. 7a, with the suggested continuation of the corona over a large range (factor 2 ) in radius down to the last stable orbit. The majority of the gravitational potential energy is dissipated in a region where there is no disc underneath, so the only seed photons for Compton cooling are a small fraction of those from the disc at larger radii. The combination of less Compton cooling and more electron heating would surely drive the coronal temperature to much higher values, leading to a much harder spectrum (as in the low/hard state). The smaller disc truncation implied by the geometry of Fig. 7c is much less of a problem, as the inner coronal flow is much smaller, so is energetically much less important.

The QPO behavior can also give some clues to the geometry, though these are somewhat ambiguous as their origin is not yet well understood. The strongest QPO is the one at low frequency (LF QPO: type C) and this increases in frequency as the spectra make a transition from the low/hard state to one of the soft states (e.g. van der Klis 2000). This indicates that the characteristic radius picked out by the variability is decreasing with decreasing strength of Comptonisation in the spectrum (e.g. di Matteo \& Psaltis 1999). Qualitatively this can be explained if the disc is truncated in the low/hard state, and that its inner edge moves inwards towards the last stable orbit during the transition. The fact that the LF QPO is not at the highest observed frequency in these spectra then indicates that the disc is still slightly truncated in these data, favouring the geometry of Fig. 7c 
over that of Fig. $7 \mathrm{~b}$ where the disc radius is already fixed at the last stable orbit.

We quantify the extent of disc truncation using the specific model of Stella, Vietri \& Morsink (1999), in which the LF QPO frequency, $\nu_{Q P O} \propto r^{-0.33}$, is set by Lense-Thirring precession of vertical perturbations of the innermost edge of the disc. The LF QPO's (Type-C) are at $2.38 \mathrm{~Hz}$ and $3.90 \mathrm{~Hz}$ for the 1st and 2nd observations respectively, while in the weak VHS data, these are found at $5.9-6.5 \mathrm{~Hz}$ as Type-B QPOs (Remillard et al. 2002). The ratio of QPO frequencies between those seen in these strong VHS, and the most weakly Comptonised VHS is 2.6 and 1.6, implying a change in radius in the relativistic precession of about a factor 1.4 and 1.2 for the first and second dataset, respectively. This assumes that the weakly comptonised VHS has disc close to the last stable orbit (KD04). These estimates from the QPO's are startlingly close to the radii derived from our coupled disc- coronal fits, which imply a disc inner radius of $\sim 1.35-1.1 \times$ and $1.3-1.1 \times$ that inferred from the high/soft state data. However, the major problem with such models is that the LF QPO is seen in the spectrum of the corona, not of the disc (van der Klis 2000; Gilfanov, Revnivtsev \& Molkov 2003; Sobolewska \& Życki 2006). The corona may be driven at this frequency by the disc, or the LF QPO might represent a mode of the corona itself, in which case there are no constraints on the geometry.

The high frequency (HF) QPO seems initially more constraining as the rapid timescale implies that it originates close to the black hole. This almost certainly arises from a resonance, as it appears as two separate features in a 3:2 ratio (see e.g. the review by Remillard 2005). If this is the parametric resonance between vertical and radial epicyclic modes of a disc in strong gravity then this requires that the HF QPO comes from the innermost stable orbit of the disc around a high spin black hole (Abramowicz \& Kluzniak 2001). This model would also require that the inner disc can be seen directly, in apparent conflict with the basic model presented here of an optically thick inner disc corona. However, we note that the HF QPO's are weak, and are not seen in our datasets (Remillard et al 2002). Instead they are detected in VHS spectra from this source where the tail is not as strong as seen here (Remillard et al 2002; KD04; Remillard 2005). It is possible that the HF QPO comes from the small fraction $\exp (-\tau)$ of emission from the disc which is able to escape directly (uncomptonised), so is absent in our data simply because these lines of sight are rarer when the corona is more optically thick and/or covers more of the inner disc. However, it is also possible that the HF QPO represents a resonance of the corona, in which case there are no visibility constraints (or constraints on black hole spin in the specific model of Blaes, Arras \& Fragile 2006).

The only observation which seems in direct conflict with all models of an optically thick inner disc corona is that of the strong, extremely broad iron line claimed for the second set of ASCA data used here (Miller et al 2004). Again, a small component, $\exp (-\tau)$ of the highly skewed line from the inner disc could be seen directly, but this is much weaker than the feature observed. Either the X-ray tail in the VHS is not formed by Comptonisation, or the spectral residuals around the iron line region do not indicate a highly relativistic line from the inner disc.

\section{ALTERNATIVES TO COMPTONISATION}

The conflict with the broad iron line motivates us to review the evidence for Comptonisation as the emission mechanism for the tail seen in these data. The only feasible alternative appears to be the jet. X-ray emission from the jet in the low/hard state has been proposed by e.g. Markoff et al (2001), so here we use this as a guide to what might be expected from these models in the VHS. Direct synchrotron emission might give rise to a power law, or broken power law, or cutoff power law tail (Markoff et al 2001; Wilms et al 2005; Markoff, Nowak \& Wilms 2005). In $\S 3$ we showed that a power law gave a completely unacceptable fit to these data, but a broken power law is as good a description of the continuum as our thermal+nonthermal Comptonisation model (see Appendix). However, such models run into difficulties when extrapolated to lower energies as the steep spectrum dominates the bolometric luminosity. Fig. 8a shows this for the best fit broken power law and disc continuum model for the second dataset. The extrapolated X-ray continuum overpredicts the simultaneously observed optical $\mathrm{V}$ and $\mathrm{B}$ points (dereddedned using $A_{v}=4.75$ ) of Jain et al (1999) by a factor of over 1000 .

Clearly the data strongly require that there is a low energy break in the continuum between the soft X-ray and optical wavebands. Such a break can be produced from jet models at the point where the jet synchrotron emission becomes optically thick to self-absorption (e.g. Markoff, Nowak \& Wilms 2005; Giannios 2005). However, there are some constrains on the energy of this break from variability arguments. The short timescale r.m.s. variability spectra of these VHS PCA data show a monotonic increase in fractional variability amplitude with energy form $3-50 \mathrm{keV}$, consistent with pivoting about a fixed point at $\sim 2 \mathrm{keV}$ (Gierliński \& Zdziarski 2005). There is no obvious reason for jet models to pick out such an energy. This contrasts with models in which the X-ray continuum is produced by Compton scattering of disc photons. This naturally produces a break in the Comptonised continuum at energies below the seed photon energy (see Fig. 8b), and since this is at $1-2 \mathrm{keV}$ there is no fine tuning required to explain this energy being picked out as the spectral pivot.

Gierliński \& Zdziarski (2005) show that for the second dataset used here, the r.m.s. variability spectrum from the PCA is well matched by thermal Compton scattering of the disc photons (see also Rodrigues et al 2004; Zdziarski et al 2005; Sobolewska \& Życki 2006 for the same results for other VHS spectra). The Comptonisation models actually underpredict the optical flux, but this is expected as the disc model here only includes direct emission, while reprocessing is likely to be an important contributor to the optical emission (e.g. Esin et al 2000; Hynes 2005), and optical synchrotron emission from the jet may also be present (see Turler et al 2004 for a model of the plateau/state C/class $\chi$ state of GRS $1915+105$ with a turndown in the optical).

Given the strength of the disc emission in the VHS then it is also rather natural to assume that this is the dominant contributor to the seed photons. Again this contrasts with the low/hard state, where the disc is much weaker, and the jet might be expected to be a more important contributor (Markoff et al 2001). However, even for the low/hard state, the jet models fail to reproduce the detailed shape of the tail 

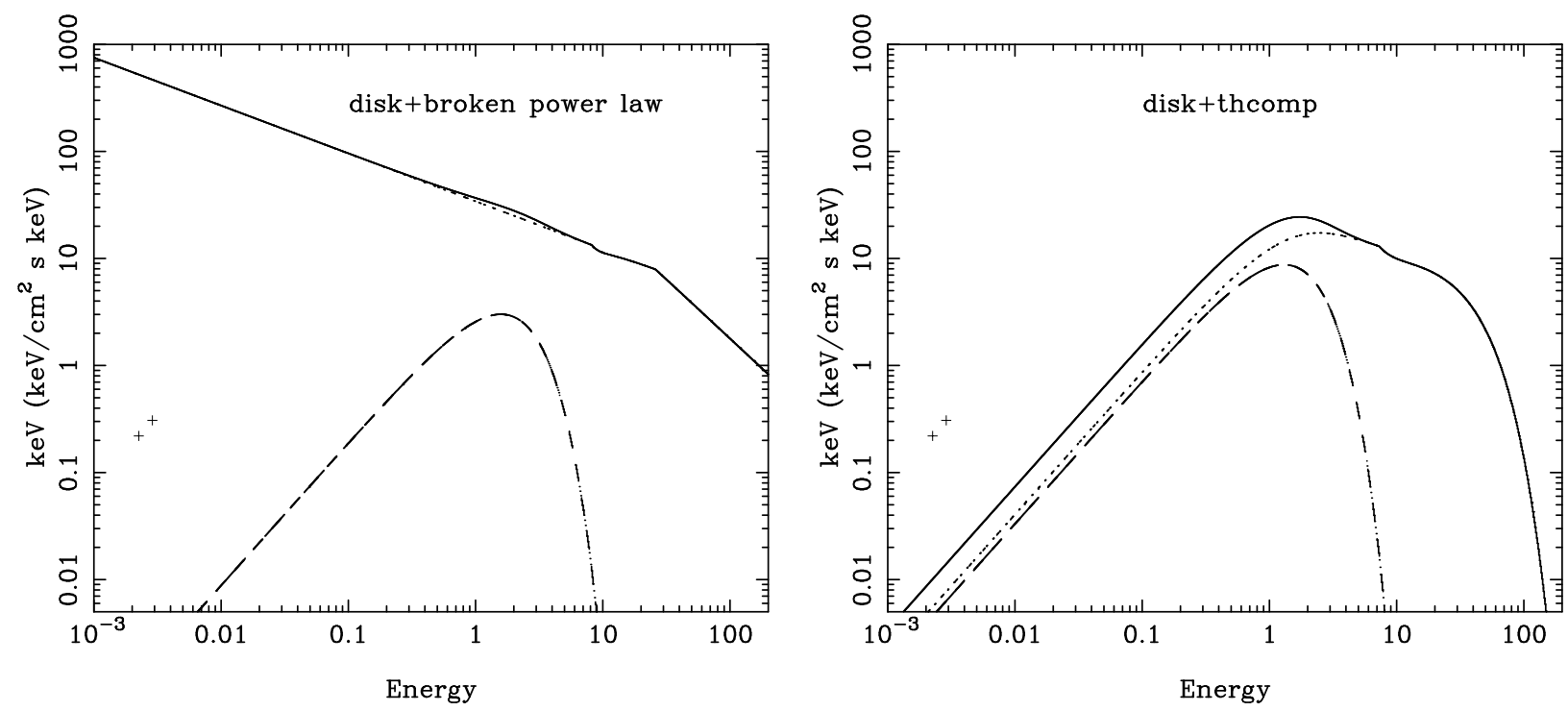

Figure 8. Absorption corrected X-ray and optical spectra of the second dataset. The left hand panel shows the best fit broken power law model for the X-ray tail. The extrapolation of this down to the optical overpredicts the simultaneously measured V and B data by a factor of over 1000. There must be a low energy break in the spectrum. This is naturally produced in Comptonisation models where the disc is the origin of the seed photons. The right hand panel shows the best fit thermal Comptonisation model for the X-ray tail. This underestimates the optical flux, leaving room for a reprocessed flux component from illumination of the disc and/or some nonthermal synchrotron emission from the outer jet.

(Zdziarski et al 2003), so that the current version of these jet models now have the majority of the X-ray emission produced by Comptonisation of the disc and cyclo-synchrotron photons by thermal electrons at the jet base (Markoff et al 2005). Since disc Comptonisation appears to be a component of the X-ray continuum even in the low/hard state, this process should be even more dominant with the stronger disc emission seen in the VHS.

Thus both the multiwavelength spectral shape and energy dependence of the X-ray variability strongly support a disc Comptonisation interpretation of the X-ray tail. We show in $\S 7$ that this is not inconsistent with the presence of high (or low) frequency QPO's. A hot (and so presumably geometrically thick) inner disc corona may actually be required to produce the strong jet (McKinney 2005) traced by radio emission during this outburst of XTE J1550 - 564 (Corbel et al 2001). The only obvious inconsistency is with the interpretation of the iron line in these data given by Miller et al (2004). However, unlike the Comptonisation interpretation of the tail, which seems to be the only viable mechanism, there does exist an alternative explanation for the line shape. Done \& Gierliński (2005) show that the ASCA spectrum in the second VHS observation used here can be fit equally well by much less extreme line smearing if there is also some ionized absorption present. The underlying iron line width is then reduced, consistent with originating from reflection in the outer disc, which allows the inner disc to be mostly covered by the corona.

Thus there are no data which unambiguously rule out an optically thick inner disc corona, while there are many observations which strongly support its existence. Such a corona will inevitably distort the disc spectrum in the ways explored in this paper.

\section{CONCLUSIONS}

We investigate the broadband continuum spectra of two VHS observations covering $0.8-200 \mathrm{keV}$. These have a strong $\mathrm{X}$-ray tail, contributing more than half of the total bolometric luminosity of the source. The disc in these spectra has an apparent temperature which is much lower than that seen from the same source at the same luminosity in the disc dominated high/soft state. We show that this is not simply produced by the effect of an optically thick corona hiding the emission from the inner disc, but instead requires that the disc changes in some way from that seen in the high/soft state, either in area (Fig. 7a), emissivity (Fig. 7b) or both (Fig. 7c).

The most obvious change in these spectra is that there is now a corona. Energetically, this must be powered ultimately by the accretion flow, so there is less power available for the disc itself. We build a specific example of disccorona coupling, where the corona over the inner disc drains power from the disc material under it, changing its emissivity (Svensson \& Zdziarski 1996). We show that this reduces the amount of disc truncation required, to a level where it is probably not significant given the model uncertainties e.g. if some fraction of the accreting mass goes through the corona as well as some fraction of the power, or if the geometry of the corona is more quasi-spherical than slab-like. However, we marginally favour the coupled disc-corona system with a slightly truncated disc (Fig. 7c) as this can simultaneously explain the LF QPO in the relativistic disc precession model. However, since there is as yet no clear consensus on models of QPO formation, this cannot give unambiguous information on disc radii. Thus Fig. 7b, where the disc area (though not its properties!) is unchanged from the disc dominated states is also consistent with the spectra, though this leaves 
the change in LF QPO as an open question. Conversely, a large truncation radius with continuous corona (Fig. 7a) is ruled out by the steep Comptonised spectrum seen.

However, we stress that irrespective of the specific model, the strong Comptonisation seen in these very high state data clearly requires that the underlying disc properties are rather different to those of a disc without a corona. Unsurprisingly, the data require that the disc structure changes in response to whatever changing dissipation mode powers the growth of the coronal structure.

We also stress that these VHS spectra are very similar to those seen from several ULX in terms of having a very low apparent disc temperature for their luminosity. This has been interpreted as evidence for intermediate mass black holes in the ULX, of order $1000 M_{\odot}$ (Miller, Fabian \& Miller 2004). Obviously this is not an option in XTE J1550 - 564, where the black hole mass is constrained by dynamical studies to be a normal stellar remnant black hole mass of order $\sim 10 M_{\odot}$ (Orosz et al 2002). We suggest that the same process, of a corona draining energy from the inner disc, may be operating in these ULX. Many of these would then be compatible with fairly normal binary systems, where the black hole mass is at the upper end of that which can reasonably form from models of stellar collapse (typically $30 M_{\odot}$ or up to $\sim 80 M_{\odot}$ from recent calculations of binary evolution; Belczynski et al. 2004) accreting at high mass accretion rates (0.1-few times the Eddington limit). We will explore this possibility in depth in a subsequent paper.

\section{ACKNOWLEDGEMENTS}

The present work is supported in part by JSPS grant of No.13304014 and No.14079101. CD thanks ISAS and RIKEN for their hospitality during visits at which this work was carried out. AK is supported by special postdoctoral researchers program in RIKEN.

\section{REFERENCES}

Abramowicz M. A., Kluzniak W, 2001, A\& A, 374, 19

Agol E., Krolik J. H., 2000, ApJ, 528, 161

Ballantyne D. R., Ross R. R., Fabian A. C., MNRAS, 2001, MNRAS, 327, 10

Blaes O., Arras P., Fragile C., 2006, MNRAS, submitted, (astro$\mathrm{ph} / 0601379)$

Belczynski K., Sadowski A., Rasio F. A., 2004, ApJ, 611, 1068

Corbel S., et al, ApJ, 554, 43

Coppi P. S., 1999, ASPC, 161, 375

di Matteo T., Psaltis D., 1999, ApJ, 526, 101

Davis S. W., Blaes O. M., Hubeny I., Turner N. J., 2005, ApJ, 621,372

Davis S. W., Done C., Blaes O. M., 2006, ApJ, in press

Done C., Gierliński M., 2003, MNRAS, 342, 1041

Done C., Madejski G. M., Życki P. T., 2000, ApJ, 536, 213

Done C., Życki P., Smith D. A., 2002, MNRAS, 331, 453

Done C., Gierliński M., 2006, MNRAS, 367, 659

Ebisawa K. et al, 1994, PASJ, 46, 375

Esin A. A., Kuulkers E., McClintock J. E., Narayan R., 2000, ApJ, 532, 1069

Giannios D., 2005, A\&A, 437, 1007

Gierliński M., Done C. 2003, MNRAS, 342, 1083

Gierliński M., Done C. 2004, MNRAS, 347, 885 (GD04)
Gierliński M., Zdziarski A. A., 2005, MNRAS, 363, 1349

Gilfanov M., Revnivtsev M., Molkov S., 2003, A\& A, 410, 217

Haardt F., Maraschi L. 1993, ApJ, 413, 507

Hynes R. I., 2005, ApJ, 623, 1026

Jain, R.K., Bailyn C.D., Orosz J.A., Remillard R.A., McClintock J.E., 1999, ApJL, 517, 131

Kubota A., et al , 1998, PASJ, 50, 667

Kubota A., Done C., 2004, MNRAS, 353, 980 (KD04)

Kubota A., Makishima K., Ebisawa K., 2001, ApJ, 560, L147

Kubota A., Makishima K., 2004, ApJ, 601, 428

Makishima K., et al 1996, PASJ, 48, 171

Markoff S., Falcke H., Fender R., 2001, A\&A, 372, L25

Markoff S., Nowak M. A., Wilms J., 2005, ApJ, 635, 1203

McClintock J. E., Remillard R. A. 2003, in Compact Stellar X-ray Sources, eds. Lewin W. H. G., \& van der Klis, M., (Cambridge University Press, Cambridge), in press (astro-ph/0306213)

McKinney J.C., 2005, ApJ, 630, 5

Merloni A., Fabian A. C., Ross R. R., 2000, MNRAS, 313, 193

Miller J. M., Fabian A. C., Miller M. C., 2004, ApJ, 614, 117

Miller J.M., et al. 2003, MNRAS, 338, 7

Miller J.M., Fabian A.C., Nowak M.A., Lewin W.H.G., 2004, to appear in proc. 10th Anual Marcel Grossmann Meeting on General Relativity (astro-ph/0402101)

Mitsuda K., et al 1984, PASJ, 36, 741

Miyamoto S., Kimura K., Kitamoto S., Dotani T., Ebisawa K. 1991, ApJ, 383, 784

Orosz J. A. et al 2002, ApJ, 568, 845

Pozdnyakov L. A., Sobol I. M., Sunyaev R. A. 1983, ASPR, 2, 189

Remillard R. A., Sobczak G. J., Muno M. P., McClintock J. E., 2002, ApJ, 564, 962

Remillard R. A., 2005, AN, 326, 804

Rodriguez J., Corbel S., Hannikainen D. C., Belloni T., Paizis A., Vilhu O., 2004, ApJ, 615, 416

Ross R. R., Fabian A. C., Young A. J., 1999, 306, 461

Shakura N., Sunyaev R., 1973, A\&A, 24, 337

Życki P. T., Sobolewska M. A., 2005, MNRAS, 364, 891

Sobczak G. J., McClintock J. E., Remillard R. A., Levine A. M., Morgan E. H, Bailyn C. D., Orosz J. A. 1999, ApJ 517, 121

Stella L., Mario V., Morsink S. M., 1999, ApJ. 524, L63

Svensson R., Zdziarski A. A., 1994, 436, 599

Tanaka Y., \& Lewin W. H. G. 1995, in X-ray Binaries, eds. Lewin W. H. G., van Paradijs J. and van den Heuvel W. P. J., (Cambridge University Press, Cambridge), p126

Türler M., Courvoisier T. J.-L., Chaty S., Fuchs Y., 2004, A\&A, 415, L35

van der Klis M., 2000, ARA\&A, 38, 717

Wilms J., Nowak M. A., Pottschmidt K., Pooley G. G., Fritz S., 2006, A\&A, 447, 245

Zdziarski A. A., Johnson W. N., Magdziarz P.,1996, MNRAS, 283,193

Zdziarski A. A., Grove J. E., Poutanen J., Rao A. R., Vadawale S. V., 2001, ApJ, 554, 45

Zdziarski A. A., Gierliński M., Rao A. R., Vadawale S. V., Mikołajewska J., 2005, MNRAS, 360, 825

Zdziarski A. A., Lubiński P., Gilfanov M., Revnivtsev M., 2003, MNRAS, 342, 355

Życki, P. T., Done, C. \& Smith, D. A., 1999, MNRAS, 305, 231

\section{APPENDIX A: DETAILS OF SPECTRAL FITS}

We use the second dataset to quantify some aspects of the fits, with a baseline model of a broken power law tail (together with disc and SEG reflection gives $\chi_{\nu}^{2}=146.3 / 193$ for $k T_{\text {disc }}=0.67_{-0.05}^{+0.04} \mathrm{keV}$. By contrast, using the newest response (PCARSP 10.1) gives $\chi_{\nu}^{2}=183.3 / 193$ for $k T_{\text {disc }}=$ 
$0.77 \pm 0.06 \mathrm{keV}$. The shift is within the uncertainties, but more important is systematic: representative high/soft state spectra also have similarly shifted temperatures so that the difference between the disc properties in the VHS and high/soft state is robust. Replacing the HEXTE data with a file without the 0.5 per cent systematic error gives $\chi_{\nu}^{2}=$ $147.6 / 193$ and $k T_{\text {disc }}=0.66_{-0.04}^{+0.05} \mathrm{keV}$, nearly identical as the statistical uncertainties are all larger than 1 per cent. 
Table A1. Best fit parameters obtained by a simultaneous ASCA/RXTE observation. The continuum is always described by a multicolour disc spectrum, plus a low temperature thermal comptonised component, together with a power law extending to high energies. The five different fits show the results of the different descriptions of reflection. These are a narrow gaussian line and smeared edge in SEG, while the other models include the full reflection spectrum (line and continuum) in the thcomp model. In the case of Xi1, Xi2 and $\mathrm{Xi} 3$, the data in $5-12 \mathrm{keV}$ is excluded from the spectral fit, $R_{\mathrm{in}}$ is fixed to $30 R_{\mathrm{g}}$, and the value of $\xi$ is fixed to $10^{2}, 10^{3}, 10^{4}$, for models Xi1, Xi2, and Xi3, respectively. Normalization factors with the PCA data are used to calculate luminosities.

\begin{tabular}{|c|c|c|c|c|c|c|c|c|c|}
\hline model & $\begin{array}{c}N_{\mathrm{H}} \\
10^{21} \mathrm{~cm}^{-1}\end{array}$ & $\begin{array}{l}T_{\text {in }} \\
\mathrm{keV}\end{array}$ & $\Gamma_{\text {thc }}$ & $\begin{array}{c}T_{\mathrm{e}} \\
\mathrm{keV}\end{array}$ & $\begin{array}{c}L_{\text {disk }} \\
\left(N_{\text {disk }}\right)\end{array}$ & $\begin{array}{l}L_{\text {thc }}(\text { slab }) \\
\left(N_{\text {thc }}\right)\end{array}$ & $L_{\text {pow }}$ & $\begin{array}{l}\text { smedge,line (a) } \\
\text { reflection (b-e) }\end{array}$ & $\chi^{2} /$ dof \\
\hline \multicolumn{10}{|c|}{ Before peak } \\
\hline SEG & $6.5 \pm 0.2$ & $0.45_{-0.01}^{+0.02}$ & $2.08_{-0.02}^{+0.01}$ & $10.0 \pm 0.5$ & $\begin{array}{c}1.03 \\
(37.6)\end{array}$ & $\begin{array}{c}3.14 \\
(58.0)\end{array}$ & 0.86 & $\begin{array}{c}E=7.9_{-0.2}^{+0.3} \mathrm{keV} \\
\tau_{\max }=0.3_{-0.1}^{+0.2} \\
\text { width }=3_{-1}^{+2} \mathrm{keV} \\
E=6.48_{-0.07}^{+0.03} \mathrm{keV} \\
\mathrm{EW}=81 \pm 10 \mathrm{eV}\end{array}$ & $201.4 / 192$ \\
\hline REF & $5.9 \pm 0.1$ & $0.51_{-0.02}^{+0.01}$ & $2.10 \pm 0.01$ & $11.6_{-0.6}^{+0.5}$ & $\begin{array}{c}0.92 \\
(30.1)\end{array}$ & $\begin{array}{c}3.05 / 2.64 \\
(51.0 / 44.9)\end{array}$ & 0.76 & $\begin{array}{l}\Omega / 2 \pi=0.23_{-0.01}^{+0.04} \\
\xi=6.8_{-2}^{+0.8} \times 10^{3} \\
R_{\text {in }}=94_{-83}^{+159} R_{\mathrm{g}}\end{array}$ & $230.2 / 194$ \\
\hline Xi1 & $6.5_{-0.3}^{+0.2}$ & $0.45 \pm 0.03$ & $2.27_{-0.03}^{+0.02}$ & $14 \pm 1$ & $\begin{array}{c}0.86 \\
(31.0)\end{array}$ & $\begin{array}{c}3.52 / 2.95 \\
(80.2 / 64.4)\end{array}$ & 0.76 & $\begin{array}{c}\Omega / 2 \pi=0.76_{-0.06}^{+0.08} \\
\left(\xi=10^{2}\right)\end{array}$ & $146.3 / 146$ \\
\hline $\mathrm{Xi} 2$ & $6.1_{-0.1}^{+0.2}$ & $0.50 \pm 0.02$ & $2.14 \pm 0.01$ & $11.5 \pm 6$ & $\begin{array}{c}0.90 \\
(29.8)\end{array}$ & $\begin{array}{l}3.14 / 2.69 \\
(57.0 / 48.5)\end{array}$ & 0.81 & $\begin{array}{c}\Omega / 2 \pi=0.37 \pm 0.07 \\
\left(\xi=10^{3}\right)\end{array}$ & $161.2 / 146$ \\
\hline $\mathrm{Xi} 3$ & $6.2 \pm 0.2$ & $0.48 \pm 0.02$ & $2.10 \pm 0.01$ & $11.3 \pm 0.6$ & $\begin{array}{c}0.97 \\
(33.5)\end{array}$ & $\begin{array}{c}3.10 / 2.41 \\
(53.5 / 42.7)\end{array}$ & 0.81 & $\begin{array}{c}\Omega / 2 \pi=0.42_{-0.04}^{+0.08} \\
\left(\xi=10^{4}\right)\end{array}$ & $157.8 / 146$ \\
\hline \multicolumn{10}{|c|}{ After peak } \\
\hline SEG & $6.7 \pm 0.2$ & $0.55 \pm 0.02$ & $2.32_{-0.03}^{+0.02}$ & $12 \pm 1$ & $\begin{array}{l}1.19 \\
(36.1)\end{array}$ & $\begin{array}{c}4.09 \\
(80.9)\end{array}$ & 0.69 & $\begin{array}{c}E=7.5 \pm 0.2 \mathrm{keV} \\
\tau_{\max }=0.6 \pm 0.2 \\
\text { width }=5_{-2}^{+3} \mathrm{keV} \\
E=6.4 \pm 0.1 \mathrm{keV} \\
\mathrm{EW}=41_{-6}^{+12} \mathrm{eV}\end{array}$ & $157.8 / 192$ \\
\hline REF & $7.1 \pm 0.1$ & $0.44_{-0.02}^{+0.06}$ & $2.74 \pm 0.03$ & $>120^{a}$ & $\begin{array}{c}0 \\
(0)\end{array}$ & $\begin{array}{c}5.87 / 5.32 \\
(163.9 / 161.9)\end{array}$ & 0.29 & $\begin{array}{c}\Omega / 2 \pi=1.7_{-0.1}^{+0.2} \\
\xi=5_{-1}^{+2} \\
R_{\text {in }}=12 \pm 2 R_{\mathrm{g}}\end{array}$ & $226.2 / 194$ \\
\hline Xi1 & $7.1_{-0.2}^{+0.3}$ & $0.53 \pm 0.03$ & $2.64 \pm 0.03$ & $38_{-9}^{+18}$ & $\begin{array}{c}0.89 \\
(27.6)\end{array}$ & $\begin{array}{c}5.20 / 4.00 \\
(139.5 / 98.5)\end{array}$ & 0.46 & $\begin{aligned} \Omega / 2 \pi & =1.6_{-0.2}^{+0.3} \\
(\xi & \left.=10^{2}\right)\end{aligned}$ & $113.5 / 146$ \\
\hline $\mathrm{Xi} 2$ & $6.5 \pm 0.3$ & $0.60 \pm 0.02$ & $2.42 \pm 0.02$ & $16 \pm 2$ & $\begin{array}{l}1.17 \\
(32.9)\end{array}$ & $\begin{array}{c}4.10 / 3.29 \\
(80.9 / 64.4)\end{array}$ & 0.62 & $\begin{array}{c}\Omega / 2 \pi=0.73_{-0.09}^{+0.05} \\
\left(\xi=10^{3}\right)\end{array}$ & $131.6 / 146$ \\
\hline $\mathrm{Xi} 3$ & $6.6_{-0.1}^{+0.3}$ & $0.55_{-0.03}^{+0.02}$ & $2.35_{-0.01}^{+0.02}$ & $14_{-1}^{+2}$ & $\begin{array}{l}1.13 \\
(34.2)\end{array}$ & $\begin{array}{c}4.18 / 3.16 \\
(80.8 / 63.1)\end{array}$ & 0.64 & $\begin{array}{c}\Omega / 2 \pi=0.62_{-0.08}^{+0.04} \\
\left(\xi=10^{4}\right)\end{array}$ & $127.9 / 146$ \\
\hline
\end{tabular}

$a$ The best fit value appeared in $>200 \mathrm{keV}$. 
Table A2. Same as Table A1 but with Dквbтh instead of DiskbB plus thсомp to describe a simple (uncoupled energetics) inner disc-corona.

\begin{tabular}{|c|c|c|c|c|c|c|c|c|c|}
\hline model & $\begin{array}{c}N_{\mathrm{H}} \\
10^{21} \mathrm{~cm}^{-1}\end{array}$ & $\begin{array}{l}T_{\text {in }} \\
\mathrm{keV}\end{array}$ & $\begin{array}{c}r_{\mathrm{in}} \\
{[\mathrm{km}]}\end{array}$ & $R_{\mathrm{c}} / r_{\mathrm{in}}$ & $\Gamma_{\text {thc }}$ & $\begin{array}{c}T_{\mathrm{e}} \\
\mathrm{keV}\end{array}$ & disk-corona $^{a}$ & $\begin{array}{l}\text { smedge } \\
\text { reflection }\end{array}$ & $\chi^{2} /$ dof \\
\hline \multicolumn{10}{|c|}{ Before peak } \\
\hline SEG & $6.6_{-0.3}^{+0.2}$ & $0.60_{-0.02}^{+0.04}$ & $129_{-18}^{+10}$ & $1.59_{-0.02}^{+0.03}$ & $2.08_{-0.02}^{+0.01}$ & $9.9_{-0.6}^{+0.4}$ & $\begin{array}{c}L=4.20 \\
L_{\text {disc }}^{\text {int }}=2.79 \\
\tau=2.3\end{array}$ & $\begin{array}{c}E=8.0_{-0.1}^{+0.2} \\
\tau_{\max }=0.29_{-0.08}^{+0.26} \\
\text { width }=2.6_{-1.6}^{+0.2} \\
E=6.48 \pm 0.07 \\
\mathrm{EW}=80 \pm 10[\mathrm{eV}]\end{array}$ & $204.9 / 192$ \\
\hline REF & $6.1 \pm 0.2$ & $0.64 \pm 0.02$ & $107_{-6}^{+5}$ & $1.58_{-0.02}^{+0.01}$ & $2.11 \pm 0.02$ & $11.8 \pm 0.4$ & $\begin{array}{c}L=4.04 \\
L_{\text {disc }}^{\text {int }}=2.48 \\
\tau=2.0\end{array}$ & $\begin{array}{c}\Omega / 2 \pi=0.32_{-0.05}^{+0.02} \\
\xi=\left(3.4_{-1.2}^{+1.5}\right) \times 10^{3} \\
R_{\text {in }}=180_{-100}^{+410}\end{array}$ & $227.3 / 194$ \\
\hline $\mathrm{Xi1}$ & $6.7 \pm 0.2$ & $0.57_{-0.04}^{+0.06}$ & $170_{-50}^{+20}$ & $1.72_{-0.04}^{+0.05}$ & $2.27 \pm 0.03$ & $14 \pm 1$ & $\begin{array}{c}L=4.32 \\
L_{\text {disc }}^{\text {int }}=3.81 \\
\tau=1.6\end{array}$ & $\Omega / 2 \pi=1.0 \pm 0.2$ & $148.9 / 146$ \\
\hline $\mathrm{Xi} 2$ & $6.2_{-0.1}^{+0.3}$ & $0.63_{-0.02}^{+0.05}$ & $110_{-15}^{+20}$ & $1.57 \pm 0.03$ & $2.12 \pm 0.01$ & $11.4_{-0.4}^{+0.5}$ & $\begin{array}{c}L=4.04 \\
L_{\text {disc }}^{\text {int }}=2.46 \\
\tau=2.0\end{array}$ & $\Omega / 2 \pi=0.48_{-0.08}^{+0.10}$ & $159.6 / 146$ \\
\hline $\mathrm{Xi3}$ & $6.1_{-0.4}^{+0.2}$ & $0.62_{-0.04}^{+0.02}$ & $110_{-7}^{+20}$ & $1.46 \pm 0.03$ & $2.094_{-0.008}^{+0.007}$ & $11.3_{-0.5}^{+0.6}$ & $\begin{array}{c}L=3.98 \\
L_{\text {disc }}^{\text {int }}=2.31 \\
\tau=2.1\end{array}$ & $\Omega / 2 \pi=0.7 \pm 0.1$ & $157.0 / 146$ \\
\hline \multicolumn{10}{|c|}{ After peak } \\
\hline SEG & $6.9 \pm 0.2$ & $0.71 \pm 0.03$ & $110_{-12}^{+6}$ & $1.70 \pm 0.05$ & $2.32_{-0.03}^{+0.02}$ & $12 \pm 1$ & $\begin{array}{c}L=5.37 \\
L_{\text {dist }}^{\text {int }}=3.97 \\
\tau=1.7\end{array}$ & $\begin{array}{c}E=7.6 \pm 0.2 \\
\tau_{\max }=0.5_{-0.2}^{+0.3} \\
\text { width }=4_{-2}^{+3} \\
E=6.39 \pm 0.08 \\
\mathrm{EW}=50 \pm 10[\mathrm{eV}]\end{array}$ & $151.1 / 192$ \\
\hline REF & $6.6 \pm 0.1$ & $0.72_{-0.05}^{+0.02}$ & $105_{-9}^{+11}$ & $1.9_{-0.1}^{+0.2}$ & $2.46_{-0.03}^{+0.08}$ & $20 \pm 1$ & $\begin{array}{c}L=5.33 \\
L_{\text {disc }}^{\text {int }}=3.83 \\
\tau=1.1\end{array}$ & $\begin{array}{c}\Omega / 2 \pi=0.64_{-0.04}^{+0.13} \\
\xi=\left(1.4_{-0.5}^{+0.3}\right) \times 10^{2} \\
R_{\text {in }}=31_{-9}^{+8}\end{array}$ & $228.5 / 194$ \\
\hline Xi1 & $6.9 \pm 0.1$ & $0.64_{-0.03}^{+0.04}$ & $130 \pm 15$ & $2.2_{-0.2}^{+0.3}$ & $2.58_{-0.03}^{+0.02}$ & $20_{-1}^{+2}$ & $\begin{array}{c}L=5.48 \\
L_{\text {disc }}^{\text {int }}=3.66 \\
\tau=1.0\end{array}$ & $\Omega / 2 \pi=1.2_{-0.4}^{+0.2}$ & $127.0 / 146$ \\
\hline Xi12 & $6.8_{-0.3}^{+0.2}$ & $0.70_{-0.05}^{+0.03}$ & $110_{-10}^{+17}$ & $1.7 \pm 0.1$ & $2.40_{-0.01}^{+0.02}$ & $16_{-1}^{+2}$ & $\begin{array}{c}L=5.35 \\
L_{\mathrm{disc}}^{\mathrm{int}}=3.75 \\
\tau=1.4\end{array}$ & $\Omega / 2 \pi=0.6 \pm 0.1$ & $121.2 / 146$ \\
\hline Xi13 & $6.8 \pm 0.2$ & $0.66 \pm 0.03$ & $120 \pm 10$ & $1.52_{-0.05}^{+0.03}$ & $2.355_{-0.012}^{0.008}$ & $16 \pm 1$ & $\begin{array}{c}L=5.37 \\
L_{\mathrm{disc}}^{\mathrm{int}}=3.47 \\
\tau=1.4\end{array}$ & $\Omega / 2 \pi=0.9_{-0.1}^{+0.2}$ & $124.2 / 146$ \\
\hline
\end{tabular}

a The unabsorbed bolometric luminosity of the Dкввтн, $L$, is calculated for inclination $70^{\circ}$ by integrating the model spectrum by $0.001-300 \mathrm{keV}$. The intrinsic disc luminosity, $L_{\mathrm{disk}}^{\mathrm{int}}$, is calculated by referring to observed $r_{\mathrm{in}}$ and $T_{\mathrm{in}}$ as $4 \pi r_{\text {in }}^{2} \sigma T_{\mathrm{in}}^{4}$. 
Table A3. Same as Table A1 but with DKвBfTh instead of DiskBв plus THсOмP to describe an inner disc-corona with coupled energetics.

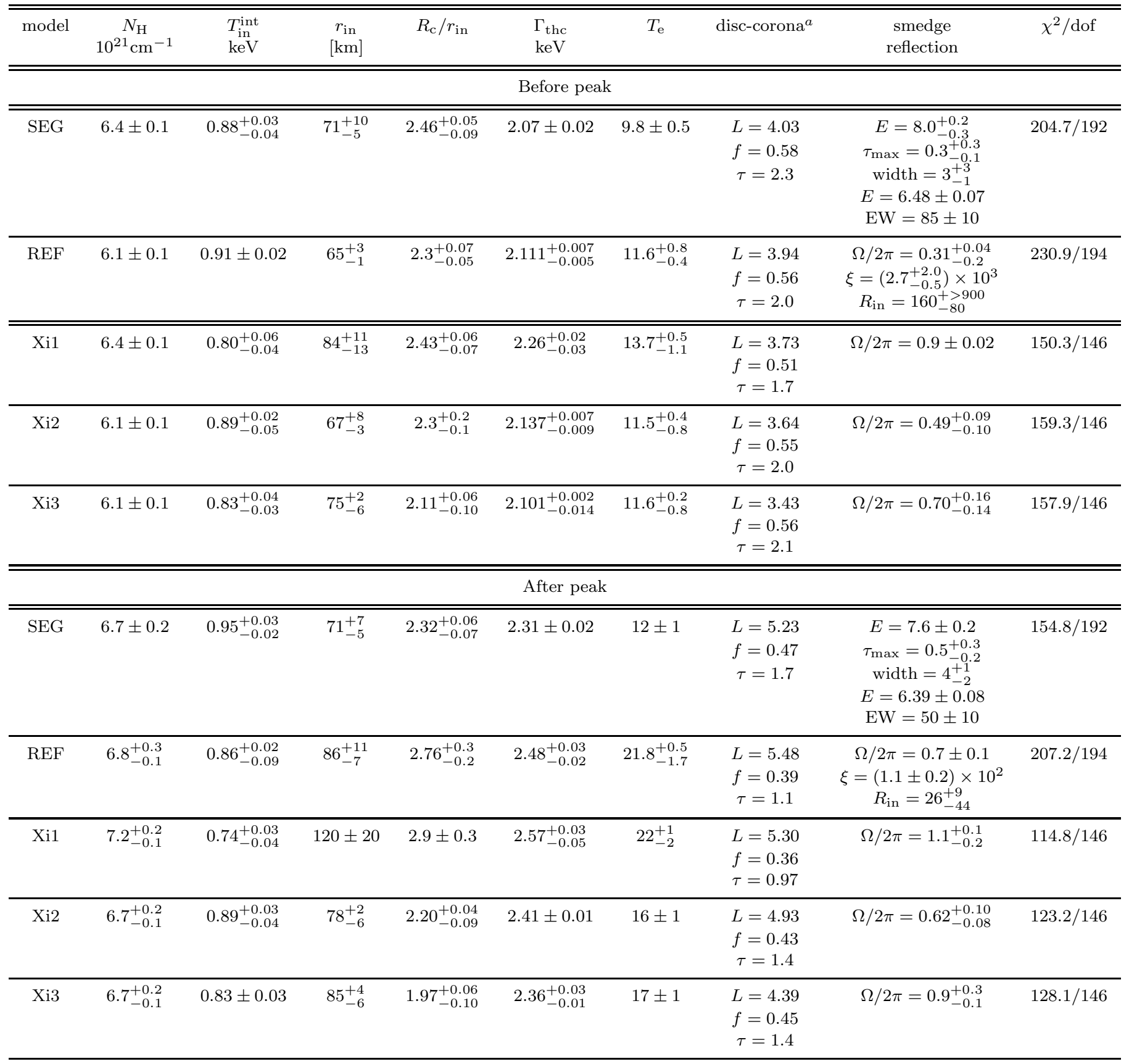

a The unabsorbed bolometric luminosity of the DкввFтн, $L$, is calculated for inclination $70^{\circ}$ by integrating the model spectrum by $0.001-300 \mathrm{keV}$. In this fit, the intrinsic disc luminosity calculated as $4 \pi r_{\mathrm{in}}^{2} \sigma T_{\mathrm{in}}^{\mathrm{int}}{ }^{4}$ 